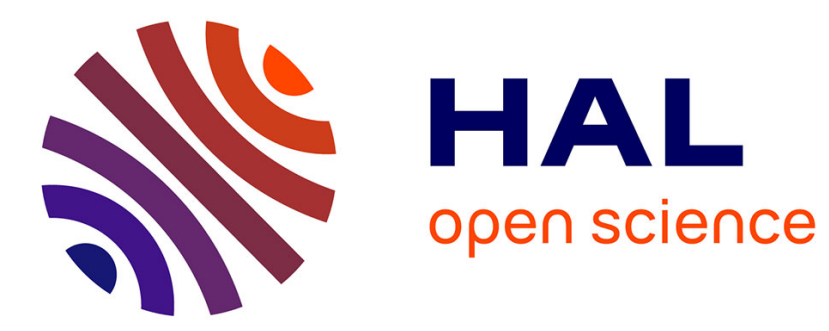

\title{
Simulation of Lévy processes and option pricing
}

El Hadj Aly Dia

\section{To cite this version:}

El Hadj Aly Dia. Simulation of Lévy processes and option pricing. The Journal of Computational Finance, 2013, 17 (é), pp.41-69. hal-00551972v4

\section{HAL Id: hal-00551972 \\ https://hal.science/hal-00551972v4}

Submitted on 28 Nov 2012

HAL is a multi-disciplinary open access archive for the deposit and dissemination of scientific research documents, whether they are published or not. The documents may come from teaching and research institutions in France or abroad, or from public or private research centers.
L'archive ouverte pluridisciplinaire HAL, est destinée au dépôt et à la diffusion de documents scientifiques de niveau recherche, publiés ou non, émanant des établissements d'enseignement et de recherche français ou étrangers, des laboratoires publics ou privés. 


\title{
SIMULATION OF LÉVY PROCESSES AND OPTION PRICING
}

\author{
EL HADJ ALY DIA*
}

\begin{abstract}
We approximate an infinite activity Lévy process by either truncating its small jumps or replacing them by a Brownian motion with the same variance. Then we derive the errors resulting from these approximations for some exotic options (Asian, barrier, lookback and American). We also propose a simple method to evaluate these options using the approximated Lévy process.
\end{abstract}

Key words. Exponential Lévy model, Exotic options, Monte-Carlo method

AMS subject classifications. 60G51, 65C05, 65N15, 60J75, 91G20

JEL classification. C02, C63, G13

1. Introduction. General exponential Lévy models (see $[2,5,11]$ ) are widely used, nowadays, in option valuation. Many numerical methods based on Fourier analysis have been subsequently developed to evaluate exotic options (see $[4,12,13,16]$ ). However, in many situations, Monte-Carlo methods have to be used, especially when the underlying Lévy process has an infinite Lévy measure. Because the simulation of such a Lévy process is not straightforward, except in some special cases (for example gamma or inverse Gaussian processes), in practice, the small jumps of the Lévy process are either truncated or replaced by a Brownian motion with the same variance. The latter approach was introduced by Asmussen and Rosinsky, who showed that, under suitable conditions, the normalized cumulated small jumps asymptotically behave like a Brownian motion (see [1]).

Many others authors were also interested in the issue of small jump approximations. We can mention Cont and Tankov (2004), Cont and Voltchkova (2005), Kohatsu-Higa and Tankov (2010), Signahl (2003) and Rydberg (1997). To our best knowledge, the behaviour of the errors resulting from these approximations for pathdependent options has not been studied yet.

The purpose of this article is to derive bounds for the errors generated by these two methods of approximation in the valuation of exotic options (Asian, barrier, lookback, and American options) in exponential Lévy models, and to propose a new method to evaluate these options.

The paper is organized as follows. In the next section, we recall some basic facts about real Lévy processes and give some useful notations. In section 3 we will study the errors resulting from the small jump approximations for Asian, barrier, lookback, and American options. The results of this section are the applications of [10]. In Section 4 we will propose a new method to evaluate exotic options when the underlying Lévy process has an infinite Lévy measure. The main idea is to reduce the valuation of these options to the simulation of the big jumps. The last section is devoted to numerical applications. We will give an example of pricing using our method and we will study the optimality of the bounds derived in Section 3.

2. Preliminaries. Consider a filtered probability space $\left(\Omega, \mathcal{F},\left(\mathcal{F}_{t}\right)_{t \in[0, T]}, \mathbb{P}\right)$.

*Université Paris-Est, Laboratoire d'Analyse et de Mathématiques Appliquées, UMR CNRS 8050, 5 bd. Descartes, Champs-sur-Marne, 77454 Marne-la-Vallée, France (dia.eha@gmail.com). 
The distribution of a Lévy process $X$ is characterized by its generating triplet $\left(\gamma, \sigma^{2}, \nu\right)$ where $(\gamma, \sigma) \in \mathbb{R} \times \mathbb{R}^{+}$, and $\nu$ is a Radon measure on $\mathbb{R} \backslash\{0\}$ satisfying

$$
\int_{\mathbb{R}}\left(1 \wedge x^{2}\right) \nu(d x)<\infty
$$

By the Lévy-Itô decomposition, $X$ can be written in the form

$$
X_{t}=\gamma t+\sigma B_{t}+\int_{|x|>1, s \in[0, t]} x J_{X}(d x \times d s)+\lim _{\delta \downarrow 0} \int_{\delta \leq|x| \leq 1, s \in[0, t]} x \widetilde{J}_{X}(d x \times d s)
$$

Here $J_{X}$ is a Poisson measure on $\mathbb{R} \times[0, \infty)$ with intensity $\nu(d x) d t, \widetilde{J}_{X}(d x \times d s)=$ $J_{X}(d x \times d s)-\nu(d x) d s$ and $B$ is a standard Brownian motion. The process $X$ has finite activity if $\nu(\mathbb{R})<\infty$. If $\nu(\mathbb{R})=+\infty$, the process $X$ is called an infinite activity Lévy process. Given $\epsilon>0$, we define the process $R^{\epsilon}$ by

$$
R_{t}^{\epsilon}=\int_{0 \leq|x| \leq \epsilon, s \in[0, t]} x \widetilde{J}_{X}(d x \times d s), t \geq 0 .
$$

Note that we have

$$
\begin{aligned}
& \mathbb{E} R_{t}^{\epsilon}=0 \\
& \operatorname{Var}\left(R_{t}^{\epsilon}\right)=\left(\sigma^{X}(\epsilon)\right)^{2} t,
\end{aligned}
$$

where

$$
\sigma^{X}(\epsilon)=\sqrt{\int_{|x| \leq \epsilon} x^{2} \nu(d x)} .
$$

The process $X^{\epsilon}$ is then defined by

$$
X_{t}^{\epsilon}=X_{t}-R_{t}^{\epsilon}, t \geq 0
$$

We also define the processes $\hat{X}^{\epsilon}$ by

$$
\hat{X}_{t}^{\epsilon}=X_{t}^{\epsilon}+\sigma(\epsilon) \hat{W}_{t}, t \geq 0,
$$

where $\hat{W}$ is a standard Brownian motion independent of $X$. Set, for any $t \geq 0$,

$$
M_{t}^{X}=\sup _{0 \leq s \leq t} X_{s}, M_{t}^{\epsilon, X}=\sup _{0 \leq s \leq t} X_{s}^{\epsilon}, \hat{M}_{t}^{\epsilon, X}=\sup _{0 \leq s \leq t} \hat{X}_{s}^{\epsilon} .
$$

The following notations will be used for the results in the next section. We define

$$
\begin{aligned}
& \sigma_{0}^{X}(\epsilon)=\max \left(\sigma^{X}(\epsilon), \epsilon\right) . \\
& \beta^{X}(\epsilon)=\frac{\int_{|x|<\epsilon} x^{4} \nu(d x)}{\left(\sigma_{0}(\epsilon)\right)^{4}} \\
& \beta_{1}^{X, t}(\epsilon)=\beta^{X}(\epsilon)^{\frac{1}{6}}\left(\sqrt{\log \left(\frac{t}{\beta(\epsilon)^{\frac{1}{3}}}+3\right)}+1\right) \\
& \beta_{p, \theta}^{X}(\epsilon)=\left(\beta^{X}(\epsilon)\right)^{\frac{p \theta}{p+4 \theta}}\left(\log \left(\frac{1}{\left(\beta^{X}(\epsilon)\right)^{\frac{p \theta}{p+4 \theta}}}\right)\right)^{p} \\
& \tilde{\beta}_{\rho, \theta}^{X}(\epsilon)=\beta_{\frac{1}{\rho}-1, \theta}^{X}(\epsilon) .
\end{aligned}
$$


When there is no ambiguity we can remove the super index $X$. Recall that Asmussen and Rosinski proved ([1], Theorem 2.1) that, if $X$ is a Lévy process, then the process $\sigma(\epsilon)^{-1} R^{\epsilon}$ converges in distribution to a standard Brownian motion, when $\epsilon \rightarrow 0$, if and only if for any $k>0$

$$
\lim _{\epsilon \rightarrow 0} \frac{\sigma(k \sigma(\epsilon) \wedge \epsilon)}{\sigma(\epsilon)}=1
$$

This result is implied by the condition

$$
\lim _{\epsilon \rightarrow 0} \frac{\sigma(\epsilon)}{\epsilon}=+\infty
$$

The conditions (2.5) and (2.6) are equivalent, if $\nu$ does not have atoms in some neighborhood of zero ([1], Proposition 2.1). On the other hand we approximate $X$ by $\hat{X}^{\epsilon}$ when the condition (2.6) is satisfied. In this case the functions $\beta, \beta_{1}, \beta_{p, \theta}$ and $\tilde{\beta}_{\rho, \theta}$ converge to 0 when $\epsilon$ goes to 0 .

REMARK 2.1. Note that, we always have $\lim _{\epsilon \rightarrow 0} \sigma(\epsilon)=0$, because all Lévy processes satisfy the condition $\int_{\mathbb{R}}\left(1 \wedge|x|^{2}\right) \nu(d x)<\infty$.

3. Error bounds for exotic options. Let $\left(S_{t}\right)_{t \in[0, T]}$ be the price of a security. The $\sigma$-algebra $\mathcal{F}_{t}$ will represent here the historical information on the price until time $t$. Under the exponential Lévy model, the process $S$ is an exponential of a Lévy process

$$
S_{t}=S_{0} e^{X_{t}}, \quad \forall t \geq 0
$$

The considered probability will be a risk-neutral probability, under which the process $\left(e^{-(r-\delta) t} S_{t}\right)_{t \in[0, T]}$ is a martingale. The parameter $r$ is the risk-free interest rate, and $\delta$ is the dividend rate. The options we will consider in the sequel will have as underlying the asset with price $S$. The option price will be denoted by $V$.

To compute $V$ by Monte Carlo methods, we need to simulate many paths of $X$. This is quite difficult, in general, for infinite activity Lévy processes. In practice we approximate $X$ by a finite activity Lévy process, by truncating its small jumps (and get $X^{\epsilon}$ ) or replacing them by a Brownian motion with the same variance (and get $\left.\hat{X}^{\epsilon}\right)$. The simulations of $X^{\epsilon}$ and $\hat{X}^{\epsilon}$ can be quite different in terms of computation time if the initial Lévy process has no Brownian component. This is why one needs to know what is the best approximation. If the condition (2.6) is satisfied, it is better to approximate $X$ by $\hat{X}^{\epsilon}$, otherwise the errors generated by the two approximations will be similar.

Set $V^{\epsilon}$ (resp. $\hat{V}^{\epsilon}$ ) as the price of the option obtained by replacing $X$ by $X^{\epsilon}$ (resp. $\left.\hat{X}^{\epsilon}\right)$. We will call $V^{\epsilon}$ (resp. $\hat{V}^{\epsilon}$ ) the approximated price by truncation (resp. the approximated price by Brownian approximation). The resulting errors will be expressed in terms of $\sigma_{0}(\epsilon)=\max (\sigma(\epsilon), \epsilon)$.

The behaviour of $\sigma(\epsilon)$ when $\epsilon$ goes to 0 depends on the Lévy measure. If there exists a real $\alpha$ such that the Lévy measure behaves like $\frac{1}{|x|^{1+\alpha}}$ when $x$ goes to 0 , then $\sigma(\epsilon)$ behaves like $\epsilon^{1-\frac{\alpha}{2}}$ when $\epsilon$ goes to 0 . This is the case for CGMY, normal inverse gaussian and varince gamma processes.

REMARK 3.1. Notice that for all infinite activity Lévy processes we have $\frac{\epsilon}{\sigma(\epsilon)}$ is bounded. 


\begin{tabular}{|c|c|c|}
\hline Call & fixed strike & floating strike \\
\hline Arithmetic mean & $\left(\frac{1}{T} \int_{0}^{T} S_{0} e^{X_{s}} d s-K\right)^{+}$ & $\left(S_{T}-\frac{1}{T} \int_{0}^{T} S_{0} e^{X_{s}} d s\right)^{+}$ \\
\hline Geometric mean & $\left(S_{0} \exp \left(\frac{1}{T} \int_{0}^{T} X_{s} d s\right)-K\right)^{+}$ & $\left(S_{T}-S_{0} \exp \left(\frac{1}{T} \int_{0}^{T} X_{s} d s\right)\right)^{+}$ \\
\hline
\end{tabular}

Payoffs of Asian call options.

\begin{tabular}{|c|c|c|}
\hline Put & fixed strike & floating strike \\
\hline Arithmetic mean & $\left(K-\frac{1}{T} \int_{0}^{T} S_{0} e^{X_{s}} d s\right)^{+}$ & $\left(\frac{1}{T} \int_{0}^{T} S_{0} e^{X_{s}} d s-S_{T}\right)^{+}$ \\
\hline Geometric mean & $\left(K-S_{0} \exp \left(\frac{1}{T} \int_{0}^{T} X_{s} d s\right)\right)^{+}$ & $\left(S_{0} \exp \left(\frac{1}{T} \int_{0}^{T} X_{s} d s\right)-S_{T}\right)^{+}$ \\
\hline
\end{tabular}

Payoffs of Asian put options.

3.1. Asian options. The payoffs of arithmetic and geometric Asian options are given in Table 3.1 and Table 3.2. We will only consider the arithmetic case. The geometric case can be deduced using the same arguments.

Proposition 3.2. Let $X$ be an infinite activity Lévy process with generating triplet $\left(\gamma, \sigma^{2}, \nu\right)$. We assume there exists $p>1$ such that $\mathbb{E} e^{p M_{T}}<\infty$. Then, the price of a continuous arithmetic Asian option with fixed strike and its approximation by truncation satisfy

$$
V=V^{\epsilon}+O\left(\sigma_{0}(\epsilon)\right)
$$

Proof. To simplifiy the proof we assume that $r=0$ and $S_{0}=1$. We have

$$
\begin{aligned}
\left|V-V^{\epsilon}\right| & \leq \mathbb{E} \frac{1}{T} \int_{0}^{T}\left|e^{X_{s}}-e^{X_{s}^{\epsilon}}\right| d s \\
& =\mathbb{E} \frac{1}{T} \int_{0}^{T}\left|X_{s}-X_{s}^{\epsilon}\right| e^{\tilde{X}_{s}^{\epsilon}} d s,
\end{aligned}
$$

where $\bar{X}_{s}^{\epsilon}$ is between $X_{s}$ and $X_{s}^{\epsilon}$. Define $p^{*}$ such that $\frac{1}{p}+\frac{1}{p^{*}}=1$. We have

$$
\begin{aligned}
\left|V-V^{\epsilon}\right| & \leq \mathbb{E} \sup _{0 \leq s \leq T}\left|R_{s}^{\epsilon}\right| \max \left(e^{M_{T}}, e^{M_{T}^{\epsilon}}\right) \\
& \leq \mathbb{E}\left(\sup _{0 \leq s \leq T}\left|R_{s}^{\epsilon}\right|^{p^{*}}\right)^{\frac{1}{p^{*}}}\left(\mathbb{E} \max \left(e^{p M_{T}}, e^{p M_{T}^{\epsilon}}\right)\right)^{\frac{1}{p}} \\
& \leq \frac{p^{*}}{p^{*}-1} \mathbb{E}\left(\left|R_{T}^{\epsilon}\right|^{p^{*}}\right)^{\frac{1}{p^{*}}}\left(\mathbb{E} e^{p M_{T}}+\mathbb{E} e^{p M_{T}^{\epsilon}}\right)^{\frac{1}{p}} \\
& =O\left(\sigma_{0}(\epsilon)\right), \text { by Proposition } 1 \text { and Lemma } 1 \text { of }[10] .
\end{aligned}
$$

$\square$

Proposition 3.3. Let $X$ be an infinite activity Lévy process with generating triplet $\left(\gamma, \sigma^{2}, \nu\right)$. We assume that there exists $p>1$ such that $\mathbb{E} e^{p M_{T}}<\infty$. Then 
the price of a continuous arithmetic Asian option with fixed strike and its Brownian approximation satisfy, for any $\theta \in(0,1)$

$$
V=\hat{V}^{\epsilon}+O\left(\sigma_{0}(\epsilon)\left(\beta_{\frac{p}{p-1}, \theta}(\epsilon)\right)^{1-\frac{1}{p}}\right) .
$$

Note that, under the condition (2.6), we have $\lim _{\epsilon \rightarrow 0} \beta_{\frac{p}{p-1}, \theta}(\epsilon)=0$.

Proof. To simplifiy the proof we assume that $r=0$ and $S_{0}=1$. Define by $f$ the payoff function of the Asian option, and set

$$
\begin{aligned}
V_{n} & =\mathbb{E} f\left(\frac{1}{n} \sum_{k=1}^{n} e^{X_{\frac{k T}{n}}}\right) \\
V_{n}^{\epsilon} & =\mathbb{E} f\left(\frac{1}{n} \sum_{k=1}^{n} e^{X_{\frac{k T}{n}}^{\epsilon}+\sigma(\epsilon) \hat{W}_{\frac{k T}{n}}}\right) .
\end{aligned}
$$

The sequence $V_{n}$ (resp. $\left.V_{n}^{\epsilon}\right)$ converges to $V\left(\right.$ resp. $\left.V^{\epsilon}\right)$. On the other hand, for $k \in\{1, \ldots, n\}$, we have

$$
R_{\frac{k t}{n}}^{\epsilon}=\frac{1}{\sqrt{n}} \sum_{j=1}^{k} V_{j}^{n},
$$

where

$$
V_{j}^{n}=\sqrt{n}\left(R_{\frac{j T}{n}}^{\epsilon}-R_{(j-1) \frac{T}{n}}^{\epsilon}\right) .
$$

The r.v. $\left(V_{j}^{n}\right)_{j \in\{1, \ldots, n\}}$ are i.i.d. and have the same distribution as $\sqrt{n} R_{\frac{t}{n}}^{\epsilon}$. But $\mathbb{E} V_{1}=0$, and $\operatorname{var}\left(V_{1}\right)=\sigma(\epsilon)^{2} t$, by Theorem 1 of [20] (see pp. 163) there exists positive i.i.d. r.v., $\left(\tau_{j}\right)_{j \in 1, \ldots, n}$, and a standard Brownian motion, $\hat{B}$, such that $\left(\sum_{j=1}^{k} V_{j}^{n}, k \in\{1, \ldots, n\}\right)$ and $\left(\hat{B}_{\tau_{1}+\cdots+\tau_{k}}, k \in\{1, \ldots, n\}\right)$ have the same joint distribution. Thus $\left(R_{\frac{k T}{n}}^{\epsilon}, k \in\{1, \ldots, n\}\right)$ and $\left(\hat{B}_{\frac{\tau_{1}+\cdots+\tau_{k}}{n}}, k \in\{1, \ldots, n\}\right)$ have the same joint distribution. We also have

$$
\left(\sigma(\epsilon) \hat{W}_{\frac{k T}{n}}, k \in\{1, \ldots, n\}\right)=^{d}\left(\hat{B}_{\frac{\sigma(\epsilon)^{2} k T}{n}}, k \in\{1, \ldots, n\}\right)
$$

Set

$$
\begin{aligned}
T_{k} & =\frac{\tau_{1}+\cdots+\tau_{k}}{n} \\
T_{k}^{\epsilon} & =\frac{\sigma(\epsilon)^{2} k T}{n} .
\end{aligned}
$$

Thus

$$
\begin{aligned}
& \sum_{k=1}^{n} e^{X_{\frac{k T}{n}}}={ }^{d} \sum_{k=1}^{n} e^{X_{\frac{k T}{n}}^{\epsilon}+\hat{B}_{T_{k}}} \\
& \sum_{k=1}^{n} e^{X_{\frac{k T}{n}}^{\epsilon}+\sigma(\epsilon) \hat{W}_{\frac{k T}{n}}}={ }^{d} \sum_{k=1}^{n} e^{X_{\frac{k T}{n}}^{\epsilon}+\hat{B}_{T_{k}^{\epsilon}}} .
\end{aligned}
$$


So

$$
\begin{aligned}
\left|V_{n}-V_{n}^{\epsilon}\right| & \leq \mathbb{E} \frac{1}{n} \sum_{k=1}^{n}\left|e^{X_{\frac{k T}{n}}^{\epsilon}+\hat{B}_{T_{k}}}-e^{X_{\frac{k T}{n}}^{\epsilon}+\hat{B}_{T_{k}^{\epsilon}}}\right| \\
& =\mathbb{E} \frac{1}{n} \sum_{k=1}^{n}\left|\hat{B}_{T_{k}}-\hat{B}_{T_{k}^{\epsilon}}\right| e^{\bar{X}_{k}^{\epsilon}}
\end{aligned}
$$

where $\bar{X}_{k}^{\epsilon}$ is between $X_{\frac{k T}{n}}^{\epsilon}+\hat{B}_{T_{k}}$ and $X_{\frac{k T}{n}}^{\epsilon}+\hat{B}_{T_{k}^{\epsilon}}$. Define $p^{*}$ such that $\frac{1}{p}+\frac{1}{p^{*}}=1$.

We have

$$
\begin{aligned}
\left|V_{n}-V_{n}^{\epsilon}\right| & \leq\left(\mathbb{E} \frac{1}{n} \sum_{k=1}^{n}\left|\hat{B}_{T_{k}}-\hat{B}_{T_{k}^{\epsilon}}\right|^{p^{*}}\right)^{\frac{1}{p^{*}}}\left(\mathbb{E} \frac{1}{n} \sum_{k=1}^{n} e^{p \bar{X}_{k}^{\epsilon}}\right)^{\frac{1}{p}} \\
& \leq\left(\mathbb{E} \sup _{1 \leq k \leq n}\left|\hat{B}_{T_{k}}-\hat{B}_{T_{k}^{\epsilon}}\right|^{p^{*}}\right)^{\frac{1}{p^{*}}}\left(\mathbb{E} \frac{1}{n} \sum_{k=1}^{n} e^{p \bar{X}_{k}^{\epsilon}}\right)^{\frac{1}{p}} .
\end{aligned}
$$

But

$$
\begin{aligned}
\mathbb{E} \frac{1}{n} \sum_{k=1}^{n} e^{p \bar{X}_{k}^{\epsilon}} & \leq \mathbb{E} \frac{1}{n} \sum_{k=1}^{n} e^{p\left(X_{\frac{k T}{n}}^{\epsilon}+\hat{B}_{T_{k}}\right)}+\mathbb{E} \frac{1}{n} \sum_{k=1}^{n} e^{p\left(X_{\frac{k T}{n}}^{\epsilon}+\hat{B}_{T_{k}^{\epsilon}}\right)} \\
& =\mathbb{E} \frac{1}{n} \sum_{k=1}^{n} e^{p\left(X_{\frac{k T}{n}}^{\epsilon}+R_{\frac{k T}{n}}^{\epsilon}\right)}+\mathbb{E} \frac{1}{n} \sum_{k=1}^{n} e^{p\left(X_{\frac{k T}{n}}^{\epsilon}+\sigma(\epsilon) \hat{W}_{\frac{k T}{n}}\right)} \\
& \leq \mathbb{E} e^{p M_{T}}+\mathbb{E} e^{p \hat{M}_{T}^{\epsilon}} \\
& \leq \mathbb{E}\left(e^{p M_{t}}+e^{p \sigma(\epsilon) \sup _{0 \leq s \leq T} \hat{W}_{s}} e^{p M_{T}^{\epsilon}}\right) \\
& \leq \mathbb{E} e^{p M_{T}}+2 e^{\frac{p^{2}}{2} \sigma(\epsilon)^{2} T} \mathbb{E} e^{p M_{T}^{\epsilon}} \\
& \leq 2 e^{\frac{p^{2}}{2} \sigma(\epsilon)^{2} T} \mathbb{E}\left(e^{p M_{T}}+e^{p M_{T}^{\epsilon}}\right)
\end{aligned}
$$

The term in the right hand side of the last inequality is bounded by a constant, say $C$, thanks to Lemma 1 of [10]. So

$$
\left|V_{n}-V_{n}^{\epsilon}\right| \leq C\left(\mathbb{E} \sup _{1 \leq k \leq n}\left|\hat{B}_{T_{k}}-\hat{B}_{T_{k}^{\epsilon}}\right|^{p^{*}}\right)^{\frac{1}{p^{*}}}
$$

Hence by Theorem 3 of [10]

$$
\left|V-V^{\epsilon}\right|=O\left(\sigma_{0}(\epsilon)\left(\beta_{\frac{p}{p-1}, \theta}(\epsilon)\right)^{1-\frac{1}{p}}\right) \quad \forall \theta \in(0,1) .
$$

The above results on fixed strike Asian options are, obviously applicable for floating strike Asian options. If the mean is geometric, we will also get the same results.

Conollary 3.4. The results of Proposition 3.2 and Proposition 3.3 are true for either arithmetic or geometric Asian options whether the strike is fixed or floating.

The proof is similar to the proofs of the above propositions. 


\begin{tabular}{|c|c|c|}
\hline Barrier & call & put \\
\hline Up Out & $\left(S_{T}-K\right)^{+} \mathbb{1}_{\left\{S_{0} e^{M_{T}<H}\right\}}$ & $\left(K-S_{T}\right)^{+} \mathbb{1}_{\left\{S_{0} e^{M_{T}}<H\right\}}$ \\
\hline Up In & $\left(S_{T}-K\right)^{+} \mathbb{1}_{\left\{S_{0} e^{M_{T}} \geq H\right\}}$ & $\left.\left(K-S_{T}\right)^{+} \mathbb{1}_{\left\{S_{0} e^{M_{T} \geq H}\right.}\right\}$ \\
\hline Down Out & $\left(S_{T}-K\right)^{+} \mathbb{1}_{\left\{S_{0} e^{m_{T}}>H\right\}}$ & $\left(K-S_{T}\right)^{+} \mathbb{1}_{\left\{S_{0} e^{m_{T}}>H\right\}}$ \\
\hline Down In & $\left(S_{T}-K\right)^{+} \mathbb{1}_{\left\{S_{0} e^{m_{T}} \leq H\right\}}$ & $\left(K-S_{T}\right)^{+} \mathbb{1}_{\left\{S_{0} e^{m_{T}} \leq H\right\}}$ \\
\hline \multicolumn{3}{|c|}{ TABLE 3.3 } \\
Payoffs of barrier options.
\end{tabular}

3.2. Barrier options. The approximation error is bigger in the barrier case compared to the other types of option. This is due to the fact that the payoff is less smooth.

Proposition 3.5. Let $X$ be an integrable Lévy process with generating triplet $\left(\gamma, \sigma^{2}, \nu\right)$. We assume that $M_{T}$ has a locally bounded probability density function. Then the price of a continuous barrier option and its approximated value by truncation satisfy, for any $q \in(0,1)$

$$
V=V^{\epsilon}+O\left(\sigma_{0}(\epsilon)^{1-q}\right)
$$

The real $q$ is used to show that the error in Proposition 3.5 is arbitrarily close to $O\left(\sigma_{0}(\epsilon)\right)$.

Proposition 3.6. Let $X$ be an integrable Lévy process with generating triplet $\left(\gamma, \sigma^{2}, \nu\right)$. We assume that $M_{T}$ has a locally bounded probability density function, then for any $\rho, \theta \in(0,1)$, the price of a continuous barrier option and its approximated value by Brownian approximation satisfy

$$
V=\hat{V}^{\epsilon}+O\left(\sigma_{0}(\epsilon)^{1-\rho}\left(\tilde{\beta}_{\rho, \theta}(\epsilon)\right)^{\rho}\right) .
$$

Here, the presence of $\rho$ and $\theta$ is due to the fact that we use Holder inequality in the proof of the intermediate result. The existence of a probability density function for $M_{T}$ and its regularity are studied in [6,9].

If the arbitrary parameter $(q)$ in Proposition 3.5 is fixed, we can get a better rate in Proposition 3.6 by chosing $\rho=q$. The reverse is not true.

Lemma 3.7. If $\mathbb{E} e^{X_{t}}<\infty$, then

$$
\mathbb{E}\left|e^{X_{t}}-e^{X_{t}^{\epsilon}}\right| \leq C \sigma_{0}(\epsilon)
$$

where $C$ is a constant independent of $\epsilon$.

Proof. We have

$$
\begin{aligned}
\mathbb{E}\left|e^{X_{t}}-e^{X_{t}^{\epsilon}}\right| & =\mathbb{E}\left|e^{X_{t}^{\epsilon}+R_{t}^{\epsilon}}-e^{X_{t}^{\epsilon}}\right| \\
& =\mathbb{E} e^{X_{t}^{\epsilon}} \mathbb{E}\left|e^{R_{t}^{\epsilon}}-1\right| \\
& \leq \mathbb{E} e^{X_{t}^{\epsilon}} \mathbb{E}\left|R_{t}^{\epsilon}\right| e^{\left(R_{t}^{\epsilon}\right)^{+}} \\
& \leq \mathbb{E} e^{X_{t}^{\epsilon}} \mathbb{E}\left|R_{t}^{\epsilon}\right|\left(e^{R_{t}^{\epsilon}}+1\right) .
\end{aligned}
$$


We can prove that for any $\beta \in \mathbb{R},\left(e^{\beta R_{t}^{\delta}}\right)_{0 \leq \delta \leq 1}$ is uniformly integrable, and if $e^{X_{s}}$ is integrable for some $s>0$, then $\left(e^{X_{t}^{\delta}}\right)_{0 \leq \delta \leq 1}$ is also uniformly integrable. Then, we use Hölder inequality and Proposition 1 of [10].

Proof of Proposition 3.5. We denote by $C$ any constant independent of $\epsilon$. We will only prove the Up and Out put case. The Down case works in the same way. For the call, the problem can be reduced to a put case by a measure change. And finally the In case can be deduced from the relation between In and Out options. We have

$$
\begin{aligned}
\left|V-V^{\epsilon}\right| \leq & \left|\mathbb{E} e^{-r T}\left(K-S_{0} e^{X_{T}}\right)^{+} \mathbb{1}_{S_{0} e^{M_{T}<H}}-\mathbb{E} e^{-r T}\left(K-S_{0} e^{X_{T}^{\epsilon}}\right)^{+} \mathbb{1}_{S_{0} e^{M_{T}^{\epsilon}<H}}\right| \\
\leq & \left|\mathbb{E} e^{-r T}\left(K-S_{0} e^{X_{T}}\right)^{+}\left(\mathbb{1}_{S_{0} e^{M_{T}<H}}-\mathbb{1}_{S_{0} e^{M_{T}^{\epsilon}<H}}\right)\right| \\
& +\left|\mathbb{E} e^{-r T}\left(\left(K-S_{0} e^{X_{T}}\right)^{+}-\left(K-S_{0} e^{X_{T}^{\epsilon}}\right)^{+}\right) \mathbb{1}_{S_{0} e^{M_{T}^{\epsilon}<H}}\right| .
\end{aligned}
$$

We define by $I_{1}$ (resp. $I_{2}$ ) the first (resp. the second) term in the right hand side of the last inequality. Set $h=\log \left(\frac{H}{S_{0}}\right)$, we have

$$
\begin{aligned}
I_{1} & =\left|\mathbb{E} e^{-r T}\left(K-S_{0} e^{X_{T}}\right)^{+}\left(\mathbb{1}_{M_{T}<h}-\mathbb{1}_{M_{T}^{\epsilon}<h}\right)\right| \\
& =\left|\mathbb{E} e^{-r T}\left(K-S_{0} e^{X_{T}}\right)^{+}\left(\mathbb{1}_{M_{T}<h, M_{T}^{\epsilon} \geq h}-\mathbb{1}_{M_{T} \geq h, M_{T}^{\epsilon}<h}\right)\right| \\
& \leq K \mathbb{P}\left[M_{T}<h, M_{T}^{\epsilon} \geq h\right]+K \mathbb{P}\left[M_{T} \geq h, M_{T}^{\epsilon}<h\right] .
\end{aligned}
$$

So, using the proof of Proposition 5 of [10], we get for any $q \in(0,1)$

$$
I_{1}=O\left(\sigma_{0}(\epsilon)^{1-q}\right) \text {. }
$$

On the other hand

$$
\begin{aligned}
I_{2} & \leq S_{0} \mathbb{E}\left|e^{X_{T}}-e^{X_{T}^{\epsilon}}\right| \\
& =O\left(\sigma_{0}(\epsilon)\right), \text { by Lemma 3.7. }
\end{aligned}
$$

Hence, for any $q \in(0,1)$

$$
\left|V-V^{\epsilon}\right|=O\left(\sigma_{0}(\epsilon)^{1-q}\right)
$$

Proof of Proposition 3.6. We consider only the Up and Out put option for the reasons mentioned in the beginning of the proof of Proposition 3.5. We set

$$
M_{T}^{n}=\sup _{0 \leq k \leq n} X_{k \frac{T}{n}}, \hat{M}_{T}^{\epsilon, n}=\sup _{0 \leq k \leq n} \hat{X}_{k \frac{T}{n}}^{\epsilon} .
$$

By the proof of Proposition 3.3, we know that

$$
\left(X_{T}, M_{T}^{n}\right)={ }^{d}\left(Y_{T}, U_{T}^{n}\right) \text { and }\left(\hat{X}_{T}^{\epsilon}, \hat{M}_{T}^{\epsilon, n}\right)={ }^{d}\left(\hat{Y}_{T}^{\epsilon}, \hat{U}_{T}^{\epsilon, n}\right),
$$


where

$$
\begin{gathered}
U_{T}^{n}=\sup _{0 \leq k \leq n}\left(X_{k \frac{T}{n}}^{\epsilon}+\hat{B}_{T_{k}}\right), Y_{T}=X_{T}^{\epsilon}+\hat{B}_{T_{n}} \\
\hat{U}_{T}^{\epsilon, n}=\sup _{0 \leq k \leq n}\left(X_{k \frac{T}{n}}^{\epsilon}+\hat{B}_{T_{k}^{\epsilon}}\right), \hat{Y}_{T}^{\epsilon}=X_{T}^{\epsilon}+\hat{B}_{T_{n}^{\epsilon}}
\end{gathered}
$$

and $\left(T_{k}\right)_{0 \leq k \leq n},\left(T_{k}^{\epsilon}\right)_{0 \leq k \leq n}$ and the Brownian motion $\hat{B}$ are defined in the proof of Proposition 3.3. So the discrete versions of $V$ and $\hat{V}$ are given by

$$
\begin{aligned}
& V^{n}=e^{-r T} \mathbb{E}\left(K-S_{0} e^{Y_{T}}\right)^{+} \mathbb{1}_{\left\{S_{0} e^{U_{T}^{n}}<H\right\}} \\
& \hat{V}^{\epsilon, n}=e^{-r T} \mathbb{E}\left(K-S_{0} e^{\hat{Y}_{T}^{\epsilon}}\right)^{+} \mathbb{1}_{\left\{S_{0} e^{\hat{U}_{T}^{\epsilon, n}}<H\right\}} .
\end{aligned}
$$

We will first study the quantity $\left|V^{n}-\hat{V}^{\epsilon, n}\right|$ as for continuous prices in the proof of Proposition 3.5, and then we use the proof of the the third result of Proposition 10 of [10] and make the limit when $n$ goes to the infinity.

3.3. Lookback and hindsight options. We will focus only on the lookback case. For the hindsight options we will use the relations between lookback and hindsight options. The quantity $S_{+}$(resp. $S_{-}$) in Table 3.4 is the predetermined maximum

\begin{tabular}{|c|c|c|}
\hline Option & call & put \\
\hline Lookback & $S_{T}-\min \left(S_{-}, S_{0} e^{m_{T}}\right)$ & $\max \left(S_{+}, S_{0} e^{M_{T}}\right)-S_{T}$ \\
\hline Hindsight & $\left(\max \left(S_{+}, S_{0} e^{M_{T}}\right)-K\right)^{+}$ & $\left(K-\min \left(S_{-}, S_{0} e^{m_{T}}\right)\right)^{+}$ \\
\hline
\end{tabular}

The payoffs of lookback and hindsight options.

(resp. minimum) of the option. We denote by $V_{l}^{c}\left(S_{-}\right)$and $V_{l}^{p}\left(S_{+}\right)$the call and the put prices of lookback options. We also denote by $V_{h}^{c}\left(S_{+}, K\right)$ and $V_{h}^{p}\left(S_{-}, K\right)$ the call and the put prices of hindsight options. So, we have the following relations between lookback and hindsight options.

$$
\begin{aligned}
V_{h}^{c}\left(S_{+}, K\right) & =V_{l}^{p}\left(\max \left(S_{+}, K\right)\right)+S_{0}-e^{-r T} K \\
V_{h}^{p}\left(S_{-}, K\right) & =V_{l}^{c}\left(\min \left(S_{-}, K\right)\right)-S_{0}+e^{-r T} K .
\end{aligned}
$$

Proposition 3.8. Let $X$ be an infinite activity Lévy process with generating triplet $\left(\gamma, \sigma^{2}, \nu\right)$. We assume that there exists $p>1$ such that $\int_{|x|>1} e^{p x} \nu(d x)<\infty$. Then the price of a continuous lookback call option and its approximation satisfy

$$
\begin{aligned}
& V\left(S_{-}\right)=V^{\epsilon}\left(S_{-}\right)+O\left(\sigma_{0}(\epsilon)\right) \\
& V\left(S_{-}\right)=\hat{V}^{\epsilon}\left(S_{-}\right)+O\left(\sigma_{0}(\epsilon) \beta_{1}^{T}(\epsilon)\right) .
\end{aligned}
$$

Proposition 3.9. Let $X$ be an infinite activity Lévy process with generating triplet $\left(\gamma, \sigma^{2}, \nu\right)$. We assume that there exists $p>1$ such that $\int_{|x|>1} e^{p|x|} \nu(d x)<\infty$. 
Then the price of a continuous lookback put option and its approximation satisfy

$$
\begin{aligned}
& V\left(S_{+}\right)=V^{\epsilon}\left(S_{+}\right)+O\left(\sigma_{0}(\epsilon)\right) \\
& V\left(S_{+}\right)=\hat{V}^{\epsilon}\left(S_{+}\right)+O\left(\sigma_{0}(\epsilon)\left(\beta_{\frac{p}{p-1}, \theta}(\epsilon)\right)^{1-\frac{1}{p}}\right) \quad \forall \theta \in(0,1) .
\end{aligned}
$$

The above results show that replacing small jumps by a Brownian motion leads to a better approximation, if the condition (2.6) is satisfied.

Proof of Proposition 3.8. To simplify the proof we assume, with no loss of generality, that $r=0$ and $S_{0}=1$. Recall that $m_{T}=-\tilde{M}_{T}$. We have

$$
\begin{aligned}
\left|V\left(S_{-}\right)-V^{\epsilon}\left(S_{-}\right)\right| & \leq \mathbb{E}\left|e^{-\tilde{M}_{T}}-e^{-\tilde{M}_{T}^{\epsilon}}\right|+\mathbb{E}\left|e^{X_{T}}-e^{X_{T}^{\epsilon}}\right| \\
& =O\left(\sigma_{0}(\epsilon)\right), \text { by Proposition } 3 \text { of [10] and Lemma 3.7. }
\end{aligned}
$$

On the other hand, we have

$$
\begin{aligned}
\left|V\left(S_{-}\right)-\hat{V}^{\epsilon}\left(S_{-}\right)\right| & \leq\left|\mathbb{E}\left(e^{-\tilde{M}_{T}}-S_{-}\right)^{+}-\mathbb{E}\left(e^{-\hat{M}_{T}^{\tilde{x}, \epsilon}}-S_{-}\right)^{+}\right|+\left|\mathbb{E}\left(e^{X_{T}}-e^{\hat{X}_{T}^{\epsilon}}\right)\right| \\
& =O\left(\sigma_{0}(\epsilon) \beta_{1}^{T}(\epsilon)\right), \text { by Theorem } 2 \text { and Proposition } 6 \text { of [10]. }
\end{aligned}
$$

Proof of Proposition 3.9. As in the proof of Proposition 3.8, we assume that $r=0$ and $S_{0}=1$. We have

$$
\begin{aligned}
\left|V\left(S_{+}\right)-V^{\epsilon}\left(S_{+}\right)\right| & \leq \mathbb{E}\left|e^{M_{T}}-e^{M_{T}^{\epsilon}}\right|+\mathbb{E}\left|e^{X_{T}}-e^{X_{T}^{\epsilon}}\right| \\
& =O\left(\sigma_{0}(\epsilon)\right), \text { by Proposition } 4 \text { of }[10] \text { and Lemma 3.7. }
\end{aligned}
$$

On the other hand, we have

$$
\begin{aligned}
\left|V\left(S_{+}\right)-\hat{V}^{\epsilon}\left(S_{+}\right)\right| & \leq\left|\mathbb{E}\left(e^{M_{T}}-S_{+}\right)^{+}-\mathbb{E}\left(e^{\hat{M}_{T}^{\epsilon}}-S_{+}\right)^{+}\right|+\left|\mathbb{E}\left(e^{X_{T}}-e^{\hat{X}_{T}^{\epsilon}}\right)\right| \\
& =O\left(\sigma_{0}(\epsilon)\left(\beta_{\frac{p}{p-1}, \theta}(\epsilon)\right)^{1-\frac{1}{p}}\right) \quad \forall \theta \in(0,1),
\end{aligned}
$$

thanks to Propositions 6 and 9 of [10].

Corollary 3.10. Propositions 3.8 and 3.9 are true for hindsight option provided that we replace, call by put, and put by call.

3.4. American options. Recall that the price of an American option with strike $K$ and maturity $T$ is given by

$$
\begin{aligned}
& \sup _{\tau \in \mathcal{T}_{[0, T]}} \mathbb{E} e^{-r \tau}\left(S_{0} e^{X_{\tau}}-K\right)^{+}, \text {for the call } \\
& \sup _{\tau \in \mathcal{T}_{[0, T]}} \mathbb{E} e^{-r \tau}\left(K-S_{0} e^{X_{\tau}}\right)^{+}, \text {for the put, }
\end{aligned}
$$

where $\mathcal{T}_{[0, T]}$ is the of stopping times with values in $[0, T]$. 
Proposition 3.11. Let $X$ be an infinite activity Lévy process with generating triplet $\left(\gamma, \sigma^{2}, \nu\right)$. Then the price of a continuous American option and its approximation by truncation satisfy

$$
V=V^{\epsilon}+O(\sigma(\epsilon)) .
$$

Proposition 3.12. Let $X$ an infinite activity Lévy process with generating triplet $\left(\gamma, \sigma^{2}, \nu\right)$. Then of a continuous American option and its Brownian approximation satisfy

$$
V=\hat{V}^{\epsilon}+O(\sigma(\epsilon))
$$

Proof of Proposition 3.11 and Proposition 3.12. For the put, we use the proof of Proposition 4.6 of [9] (pp. 67-68). Furthermore, using the proof of Proposition 3.5, the call price is given by

$$
\sup _{\tau \in \mathcal{T}_{[0, T]}} e^{-r \tau} \mathbb{E} e^{X_{\tau}} \overline{\mathbb{E}}\left(S_{0}-K e^{\tilde{X}_{\tau}}\right)^{+},
$$

where $\overline{\mathbb{E}}$ is the expectation under $\overline{\mathbb{P}}$, an equivalent measure of $\mathbb{P}$ which comes from the Esscher transform with Radon-Nikodym derivative

$$
\left.\frac{d \overline{\mathbb{P}}}{d \mathbb{P}}\right|_{\mathcal{F}_{t}}=\frac{e^{X_{t}}}{\mathbb{E} e^{X_{t}}} .
$$

We will denote by $\left(\bar{\gamma}, \bar{\sigma}^{2}, \bar{\nu}\right)$ the generating triplet of $\tilde{X}$ under $\bar{P}$. In particular we have

$$
\bar{\nu}(d x)=e^{-x} \nu(-d x) .
$$

See Section 9.4 and Section 9.5 of [7] for more details. Hence

$$
\begin{aligned}
\left|V-\hat{V}^{\epsilon}\right|= & \left|\sup _{\tau \in \mathcal{T}_{[0, T]}} e^{-r \tau} \mathbb{E} e^{X_{\tau}} \overline{\mathbb{E}}\left(S_{0}-K e^{\tilde{X}_{\tau}}\right)^{+}-\sup _{\tau \in \mathcal{T}_{[0, T]}} e^{-r \tau} \mathbb{E} e^{X_{\tau}^{\epsilon}} \overline{\mathbb{E}}\left(S_{0}-K e^{\tilde{X}_{\tau}^{\epsilon}}\right)^{+}\right| \\
= & \left|\sup _{\tau \in \mathcal{T}_{[0, T]}} e^{-r \tau} \mathbb{E} e^{X_{\tau}} \overline{\mathbb{E}}\left(S_{0}-K e^{\tilde{X}_{\tau}^{\epsilon}}\right)^{+}-\sup _{\tau \in \mathcal{T}_{[0, T]}} e^{-r \tau} \mathbb{E} e^{X_{\tau}^{\epsilon}} \overline{\mathbb{E}}\left(S_{0}-K e^{\tilde{X}_{\tau}^{\epsilon}}\right)^{+}\right| \\
& +\left|\sup _{\tau \in \mathcal{T}_{[0, T]}} e^{-r \tau} \mathbb{E} e^{X_{\tau}} \overline{\mathbb{E}}\left(S_{0}-K e^{\tilde{X}_{\tau}}\right)^{+}-\sup _{\tau \in \mathcal{T}_{[0, T]}} e^{-r \tau} \mathbb{E} e^{X_{\tau}} \overline{\mathbb{E}}\left(S_{0}-K e^{\tilde{X}_{\tau}^{\epsilon}}\right)^{+}\right| .
\end{aligned}
$$

Note by $I_{1}$ (resp. $I_{2}$ ) the first (resp. the second) term in the right hand side of the last equality. Set

$$
\begin{aligned}
& I_{1}^{1}=\sup _{\tau \in \mathcal{T}_{[0, T]}} e^{-r \tau}\left(\mathbb{E} e^{X_{\tau}}-\mathbb{E} e^{X_{\tau}^{\epsilon}}\right) \overline{\mathbb{E}}\left(S_{0}-K e^{\tilde{X}_{\tau}^{\epsilon}}\right)^{+} \\
& I_{1}^{2}=\sup _{\tau \in \mathcal{T}_{[0, T]}} e^{-r \tau}\left(\mathbb{E} e^{X_{\tau}^{\epsilon}}-\mathbb{E} e^{X_{\tau}}\right) \overline{\mathbb{E}}\left(S_{0}-K e^{\tilde{X}_{\tau}^{\epsilon}}\right)^{+} .
\end{aligned}
$$


We can show that

$$
\left|I_{1}\right| \leq\left|\max \left(I_{1}^{1}, I_{1}^{2}\right)\right|
$$

Using the proof of Proposition 4.3 of [9], we get

$$
I_{1}=O\left(\sigma(\epsilon)^{2}\right)
$$

Furthermore by Remark 2 of [10], we have

$$
I_{2}=O(\sigma(\epsilon)) .
$$

This proves the second result of Proposition 3.11. For the second result of Proposition 3.12, we use the same arguments, but we use this time the proof of Proposition 4.3 of [9] by replacing $X^{\epsilon}$ by $\hat{X}^{\epsilon}$ and Remark 2 of [10].

4. Simulation of infinite activity Lévy processes. In this section we will show a new method to simulate an infinite activity Lévy process. The first step is to approximate the Lévy process by a finite activity Lévy process as seen above. Then we will simulate the big jumps. Not that others methods of simulation of Lévy processes are proposed in $[7,21,22]$.

We have (see Section 2)

$$
\begin{aligned}
X_{t}^{\epsilon}= & X_{t}-R_{t}^{\epsilon} \\
= & \gamma t+\int_{|x|>1, s \in[0, t]} x J_{X}(d x \times d s)+\int_{\epsilon \leq|x| \leq 1, s \in[0, t]} x \tilde{J}_{X}(d x \times d s) \\
= & \left(\gamma-\int_{\epsilon \leq|x| \leq 1} x \nu(d x)\right) t+\int_{|x|>\epsilon, s \in[0, t]} x J_{X}(d x \times d s) \\
= & \left(\gamma-\int_{\epsilon \leq|x| \leq 1} x \nu(d x)\right) t+\int_{x>\epsilon, s \in[0, t]} x J_{X}(d x \times d s) \\
& +\int_{x<-\epsilon, s \in[0, t]} x J_{X}(d x \times d s) \\
= & \gamma_{0}^{\epsilon} t+\sum_{i=1}^{N_{t}^{+}} Y_{i}^{+}-\sum_{i=1}^{N_{t}^{-}} Y_{i}^{-},
\end{aligned}
$$

where

$$
\gamma_{0}^{\epsilon}=\gamma-\int_{\epsilon \leq|x| \leq 1} x \nu(d x)
$$

The r.v. $\left(Y_{i}^{+}\right)_{i \geq 1}$ are i.i.d. with common law $\frac{\nu_{\epsilon}^{+}(d x)}{\nu(\epsilon,+\infty)}$, the r.v. $\left(Y_{i}^{-}\right)_{i \geq 1}$ are i.i.d. with common law $\frac{\nu_{\epsilon}^{-}(-d x)}{\nu(-\infty, \epsilon)}$. The measures $\nu_{\epsilon}^{+}$and $\nu_{\epsilon}^{-}$correspond respectively to $\nu$ restricted to $(0,+\infty)$ and $(-\infty, 0)$. The process $X^{\epsilon}$ is a compound Poisson process.

4.1. Valuation of barrier and lookback options. Since the supremum of a compound Poisson process is reached at its jump times or its extremities, we can simulate the supremum of $X^{\epsilon}$ without any discretization. So we can compute the barrier and lookback options without discretizing the time axis. When we replace $X$ by $\hat{X}^{\epsilon}$, to evaluate barrier option we can use Example 6.1 of [7] to avoid time 
discretization. For lookback options, we can use the jump times as discretization points of the Brownian motion. So, the supremum and the infimum of $\hat{X}^{\epsilon}$ should be replaced by

$$
\begin{aligned}
\bar{M}_{T}^{\epsilon} & =\max _{0 \leq j \leq N_{t}^{\epsilon}+1} \hat{X}_{\hat{T}_{j}^{\epsilon}} \\
\bar{m}_{T}^{\epsilon} & =\min _{0 \leq j \leq N_{t}^{\epsilon}+1} \hat{X}_{\hat{T}_{j}^{\epsilon}},
\end{aligned}
$$

where

$$
\begin{aligned}
& \hat{T}_{0}^{\epsilon}=0 \\
& \hat{T}_{j}^{\epsilon}=T_{j}^{\epsilon} \wedge T,
\end{aligned}
$$

and $\left(T_{j}^{\epsilon}\right)_{j \geq 1}$ are the jump times of $X^{\epsilon}$. We will denote by $\bar{V}^{\epsilon}$ the corresponding price of the lookback option.

Proposition 4.1. Let $X$ be an infinite activity Lévy process with generating triplet $(\gamma, 0, \nu)$. We assume that there exists $p>1$ such that $\int_{|x|>1} e^{p x} \nu(d x)<\infty$. Then

$$
\hat{V}^{\epsilon}=\bar{V}^{\epsilon}+o(\sigma(\epsilon)),
$$

where $\hat{V}^{\epsilon}$ denotes the price of the lookback option where $X$ is replaced by $\hat{X}^{\epsilon}$. The condition $\int_{|x|>1} e^{p x} \nu(d x)<\infty$ is necessary only for the put.

Proof. We will only prove the put case (the call case is quite easy). We have

$$
\begin{aligned}
\left|\hat{V}^{\epsilon}-\bar{V}^{\epsilon}\right| & \leq S_{0} \mathbb{E}\left|e^{\hat{M}_{T}^{\epsilon}}-e^{\bar{M}_{T}^{\epsilon}}\right| \\
& \leq S_{0} \mathbb{E} e^{\max \left(\hat{M}_{T}^{\epsilon}, \bar{M}_{T}\right)}\left|\hat{M}_{T}^{\epsilon}-\bar{M}_{T}^{\epsilon}\right| .
\end{aligned}
$$

So using Cauchy-Schwarz inequality, we get

$$
\left|\hat{V}^{\epsilon}-\bar{V}^{\epsilon}\right| \leq S_{0}\left(\mathbb{E} e^{p \max \left(\hat{M}_{T}, \bar{M}_{T}\right)}\right)^{\frac{1}{p}}\left(\mathbb{E}\left|\hat{M}_{T}-\bar{M}_{T}\right|^{\frac{p}{p-1}}\right)^{\frac{p-1}{p}}
$$

Note that, we have

$$
\bar{M}_{T}^{\epsilon} \leq \hat{M}_{T}^{\epsilon}
$$

On the other hand

$$
\begin{aligned}
\hat{M}_{T}^{\epsilon} & =\sup _{0 \leq s \leq T}\left(\sigma(\epsilon) \hat{W}_{s}+X_{s}^{\epsilon}\right) \\
& \leq \sigma(\epsilon) \sup _{0 \leq s \leq T} \hat{W}_{s}+M_{T}^{\epsilon} .
\end{aligned}
$$

So

$$
\mathbb{E} e^{p \max \left(\hat{M}_{T}, \bar{M}_{T}\right)} \leq 2 e^{\frac{p^{2} \sigma(\epsilon)^{2} T}{2}} \mathbb{E} e^{p M_{T}^{\epsilon}}
$$

Hence, using Lemma 1 of [10], there exists a constant $C>0$ (independent of $\epsilon$ ) such that

$$
\left|\hat{V}^{\epsilon}-\bar{V}^{\epsilon}\right| \leq C\left(\mathbb{E}\left|\hat{M}_{T}^{\epsilon}-\bar{M}_{T}^{\epsilon}\right|^{\frac{p}{p-1}}\right)^{\frac{p-1}{p}}
$$


The following lemma concludes the proof. $\square$

Lemma 4.2. For any $p \geq 1$

$$
\mathbb{E}\left|\hat{M}_{T}^{\epsilon}-\bar{M}_{T}^{\epsilon}\right|^{p}=o\left((\sigma(\epsilon))^{p}\right) .
$$

The proof of Lemma 4.2 can be found in the appendix.

4.2. Valuation of Asian options. We will focus on the fixed strike Asian put option. The call case can be easily deduced. Floating Asian options, can be evaluated using fixed strike options and symmetry. Consider the following payoffs

$$
\begin{aligned}
& \left(K-\frac{1}{T} \int_{0}^{T} S_{0} e^{X_{s}} d s\right)^{+}, \text {arithmetic Asian put } \\
& \left(K-S_{0} e^{\frac{1}{T} \int_{0}^{T} X_{s} d s}\right)^{+}, \text {geometric Asian put. }
\end{aligned}
$$

We set

$$
\begin{aligned}
& V_{a}=e^{-r T} \mathbb{E}\left(K-\frac{1}{T} \int_{0}^{T} S_{0} e^{X_{s}} d s\right)^{+} \\
& V_{g}=e^{-r T} \mathbb{E}\left(K-S_{0} e^{\frac{1}{T} \int_{0}^{T} X_{s} d s}\right)^{+} .
\end{aligned}
$$

The Lévy process $X$ considered has the generating triplet $(\gamma, 0, \nu)$. In fact we will estimate the quantities $V_{a}^{\epsilon}, \hat{V}_{a}^{\epsilon}, V_{g}^{\epsilon}$ and $\hat{V}_{g}^{\epsilon}$ obtained by replacing $X$ by $X^{\epsilon}$ or $\hat{X}^{\epsilon}$.

We have

$$
\begin{aligned}
V_{a}^{\epsilon} & =e^{-r T} \mathbb{E}\left(K-\frac{1}{T} \int_{0}^{T} S_{0} e^{X_{s}^{\epsilon}} d s\right)^{+} \\
& =e^{-r T} \mathbb{E}\left(K-\frac{S_{0}}{T} \sum_{j=1}^{N_{T}^{\epsilon}+1} \int_{\hat{T}_{j-1}^{\epsilon}}^{\hat{T}_{j}^{\epsilon}} e^{X_{s}^{\epsilon}} d s\right)^{+} \\
& =e^{-r T} \mathbb{E}\left(K-\frac{S_{0}}{T} \sum_{j=1}^{N_{T}^{\epsilon}+1} \int_{\hat{T}_{j-1}^{\epsilon}}^{\hat{T}_{j}^{\epsilon}} e^{\gamma_{0}^{\epsilon} s+\sum_{i=1}^{j-1} Y_{i}^{\epsilon}} d s\right)^{+}, \text {see }(4.1) .
\end{aligned}
$$

So

$$
\begin{aligned}
V_{a}^{\epsilon} & =e^{-r T} \mathbb{E}\left(K-\frac{S_{0}}{T} \sum_{j=1}^{N_{T}^{\epsilon}+1} e^{\sum_{i=1}^{j-1} Y_{i}^{\epsilon}} \int_{\hat{T}_{j-1}^{\epsilon}}^{\hat{T}_{j}^{\epsilon}} e^{\gamma_{0}^{\epsilon} s} d s\right)^{+} \\
& =e^{-r T} \mathbb{E}\left(K-\frac{S_{0}}{T} \sum_{j=1}^{N_{T}^{\epsilon}+1} e^{\sum_{i=1}^{j-1} Y_{i}^{\epsilon}} \frac{e^{\gamma_{0}^{\epsilon} \hat{T}_{j}^{\epsilon}}-e^{\gamma_{0}^{\epsilon} \hat{T}_{j-1}^{\epsilon}}}{\gamma_{0}^{\epsilon}}\right)^{+} \\
& =e^{-r T} \mathbb{E}\left(K-\frac{S_{0}}{T} \sum_{j=1}^{N_{T}^{\epsilon}+1} \frac{e^{\gamma_{0}^{\epsilon} \hat{T}_{j}^{\epsilon}+\sum_{i=1}^{j-1} Y_{i}^{\epsilon}}-e^{\gamma_{0}^{\epsilon} \hat{T}_{j-1}^{\epsilon}+\sum_{i=1}^{j-1} Y_{i}^{\epsilon}}}{\gamma_{0}^{\epsilon}}\right)^{+}
\end{aligned}
$$


Hence

$$
V_{a}^{\epsilon}=e^{-r T} \mathbb{E}\left(K-\frac{S_{0}}{T} \sum_{j=1}^{N_{T}^{\epsilon}+1} \frac{e^{X_{\hat{T}_{j}^{\epsilon-}}^{\epsilon}}-e^{X_{\hat{T}_{j-1}^{\epsilon}}^{\epsilon}}}{\gamma_{0}^{\epsilon}}\right)^{+}
$$

By the same way, we get

$$
V_{g}^{\epsilon}=e^{-r T} \mathbb{E}\left(K-S_{0} \exp \left(\frac{1}{T} \sum_{j=1}^{N_{T}^{\epsilon}+1} \sum_{i=1}^{j-1} Y_{i}^{\epsilon}+\frac{1}{2}\left(\left(\hat{T}_{j}^{\epsilon}\right)^{2}-\left(\hat{T}_{j-1}^{\epsilon}\right)^{2}\right)\right)\right)^{+}
$$

In geometric case when we replace $X^{\epsilon}$ by $\hat{X}^{\epsilon}$, we get

$$
\begin{aligned}
\hat{V}_{g}^{\epsilon}=e^{-r T} \mathbb{E} & \left(K-S_{0} \exp \left(\frac{1}{T} \sum_{j=1}^{N_{T}^{\epsilon}+1} \sum_{i=1}^{j-1} Y_{i}^{\epsilon}+\frac{1}{2}\left(\left(\hat{T}_{j}^{\epsilon}\right)^{2}-\left(\hat{T}_{j-1}^{\epsilon}\right)^{2}\right)\right.\right. \\
+ & \left.\left.\sigma(\epsilon) \int_{\hat{T}_{j-1}^{\epsilon}}^{\hat{T}_{j}^{\epsilon}} \hat{W}_{s} d s\right)\right)^{+} .
\end{aligned}
$$

And given $N_{T}^{\epsilon}$ and $\left(\hat{T}_{j}^{\epsilon}\right)_{1 \leq j \leq N_{T}^{\epsilon}}$, the r.v. $\left(\int_{\hat{T}_{j-1}^{\epsilon}}^{\hat{T}_{j}^{\epsilon}} \hat{W}_{s} d s\right)_{1 \leq j \leq N_{T}^{\epsilon}+1}$ are independent with Gaussian distribution with mean 0 and variance $\frac{1}{3}\left(\hat{T}_{j}^{\epsilon}-\hat{T}_{j-1}^{\epsilon}\right)^{3}$. In the arithmetic case, we have the following result.

Proposition 4.3. Let $X$ be an infinite activity Lévy process with generating triplet $(\gamma, 0, \nu)$ and $f$ be a Lipschitz function. We assume that $\mathbb{E} e^{M_{T}}<\infty$. Then

$$
\begin{aligned}
\mathbb{E} f\left(\frac{1}{T} \int_{0}^{T} S_{0} e^{\hat{X}_{s}^{\epsilon}} d s\right)= & \mathbb{E} f\left(\frac{S_{0}}{T} \sum_{j=1}^{N_{T}^{\epsilon}+1} e^{\hat{X}_{\hat{T}_{j-1}^{\epsilon}}^{\epsilon}}\left(\frac{e^{\gamma_{0}^{\epsilon}\left(\hat{T}_{j}^{\epsilon}-\hat{T}_{j-1}^{\epsilon}\right)}-1}{\gamma_{0}^{\epsilon}}+\sigma(\epsilon) g_{j}^{\epsilon}\right)\right) \\
& +O\left(\sigma(\epsilon)^{2}\right)
\end{aligned}
$$

with

$$
g_{j}^{\epsilon}=\int_{\hat{T}_{j-1}^{\epsilon}}^{\hat{T}_{j}^{\epsilon}} e^{\gamma_{0}^{\epsilon}\left(s-\hat{T}_{j-1}^{\epsilon}\right)}\left(\hat{W}_{s}-\hat{W}_{\hat{T}_{j-1}^{\epsilon}}\right) d s
$$
and

Given $N_{T}^{\epsilon}$ and $\left(\hat{T}_{j}^{\epsilon}\right)_{1 \leq j \leq N_{T}^{\epsilon}}$, the r.v. $\left(g_{j}^{\epsilon}\right)_{1 \leq j \leq N_{T}^{\epsilon}+1}$ are independent and gaussian,

$$
\operatorname{var}\left(g_{j}^{\epsilon}\right)=\frac{1}{2\left(\gamma_{0}^{\epsilon}\right)^{3}}\left(\left(2 \gamma_{0}^{\epsilon}\left(\hat{T}_{j}^{\epsilon}-\hat{T}_{j-1}^{\epsilon}\right)-3\right) e^{2 \gamma_{0}^{\epsilon}\left(\hat{T}_{j}^{\epsilon}-\hat{T}_{j-1}^{\epsilon}\right)}+4 e^{\gamma_{0}^{\epsilon}\left(\hat{T}_{j}^{\epsilon}-\hat{T}_{j-1}^{\epsilon}\right)}-1\right)
$$

However $g_{j}^{\epsilon}$ is correlated with $\hat{W}_{\hat{T}_{j}^{\epsilon}}-\hat{W}_{\hat{T}_{j-1}^{\epsilon}}$. Indeed, we have

$$
\operatorname{cov}\left(g_{j}^{\epsilon}, \hat{W}_{\hat{T}_{j}^{\epsilon}}-\hat{W}_{\hat{T}_{j-1}^{\epsilon}}\right)=\frac{\hat{T}_{j}^{\epsilon}-\hat{T}_{j-1}^{\epsilon}}{\gamma_{0}^{\epsilon}} e^{\gamma_{0}^{\epsilon}\left(\hat{T}_{j}^{\epsilon}-\hat{T}_{j-1}^{\epsilon}\right)}-\frac{e^{\gamma_{0}^{\epsilon}\left(\hat{T}_{j}^{\epsilon}-\hat{T}_{j-1}^{\epsilon}\right)}-1}{\left(\gamma_{0}^{\epsilon}\right)^{2}}
$$


A natural control variate for $\hat{V}_{a}^{\epsilon}\left(\right.$ resp. $\left.V_{a}^{\epsilon}\right)$ is $\frac{1}{T} \int_{0}^{T} S_{0} e^{\hat{X}_{s}^{\epsilon}} d s\left(\right.$ resp. $\left.\frac{1}{T} \int_{0}^{T} S_{0} e^{X_{s}^{\epsilon}} d s\right)$. We must approximate their values.

Proposition 4.4. Let $X$ be an infinite activity Lévy process with generating triplet $\left(\gamma, \sigma^{2}, \nu\right)$ and $f$ a Lipschitz function. We assume that $\int_{|x|>1} e^{x} \nu(d x)<\infty$. Then we have

$$
\begin{aligned}
& \mathbb{E} \int_{0}^{T} e^{X_{s}} d s-\mathbb{E} \int_{0}^{T} e^{X_{s}^{\epsilon}} d s=\frac{\sigma(\epsilon)^{2}}{2} \int_{0}^{T} s \mathbb{E} e^{X_{s}^{\epsilon}} d s+O\left(\sigma_{0}(\epsilon)^{3}\right) \\
& \mathbb{E} \int_{0}^{T} e^{X_{s}} d s-\mathbb{E} \int_{0}^{T} e^{\hat{X}_{s}^{\epsilon}} d s=O\left(\sigma_{0}(\epsilon)^{3}\right) .
\end{aligned}
$$

Recall that in the case where we replace $X_{s}$ by $X_{s}^{\epsilon}$, the approximation error for Asian option is $O\left(\sigma_{0}(\epsilon)\right)$. So we do not need to know the term $\int_{0}^{T} s \mathbb{E} e^{X_{s}^{\epsilon}} d s$. Furthermore under the condition $\left(e^{(r-\delta) s+X_{s}}\right)_{s \geq 0}$ is a martingale, we have

$$
\mathbb{E} \int_{0}^{T} e^{X_{s}} d s=\left\{\begin{array}{lr}
\frac{e^{(r-\delta) T}-1}{r-\delta} & \text { if } r-\delta \neq 0 \\
T & \text { otherwise }
\end{array}\right.
$$

The proofs of Proposition 4.4 and Proposition 4.3 can be found in the appendix.

4.3. Simulation of big jumps. The simulation of the jump times is relatively simple. The problem is, because the numbers of jumps on $[0, T]$ can be large, how to quickly simulate the size of the jumps. We will focus on the simulation of the positive jumps $\left(Y_{i}^{+}\right)_{i>1}$. The simulation of $\left(Y_{i}^{-}\right)_{i>1}$ will be similar. Let $\lambda_{+}^{\epsilon}=\nu(\epsilon, \infty)$. The cumulative distribution function (cdf) of $Y_{1}^{+}$, and its inverse, cannot be determined explicitly. So one way to simulate $Y_{1}^{+}$is to use rejection sampling. This can be time consuming for some Lévy measure which have a singularity at 0 , especially since it will make on average $\lambda_{+}^{\epsilon} T$ simulations. For CGMY processes Cont and Tankov (2004) proposed a method based on rejection sampling.

The alternative is to make an inversion of the cdf, $F_{+}$, of $Y_{1}^{+}$. The method proposed by Taufer and Leonenko (2009) can be used to achieve this issue, if $Y_{1}^{+}$is self-decomposable (see [21] for more details).

We propose an other way to invert $F_{+}$. This method, though introduces a new error in the simulations, has the advantage to be simple and easily implementatble. We have, for all $x>\epsilon$

$$
F_{+}(x)=\frac{1}{\lambda_{+}^{\epsilon}} \int_{\epsilon}^{x} \nu(d x)
$$

Define a positive real $A$ in order to have $\nu(A,+\infty)$ very small, of order of $10^{-16}$ for example (this is what we use in our simulations). We suppose then that the r.v. $Y_{1}^{+}$ is in $[\epsilon, A]$. Set for any $k \in\{0, \ldots, n\}$

$$
\begin{aligned}
& x_{k}=k \frac{A-\epsilon}{n}+\epsilon \\
& y_{k}=\frac{F_{+}\left(x_{k}\right)}{F_{+}(A)} .
\end{aligned}
$$


Where $n$ is the number of the discretization points on $[\epsilon, A]$. Note that $y_{0}=0$. How do we compute $\left(F_{+}\left(x_{k}\right)\right)_{1 \leq k \leq n}$ ? Notice that for any $k \in\{1, \ldots, n\}$, we have

$$
F_{+}\left(x_{k}\right)=\sum_{j=1}^{k}\left(F_{+}\left(x_{j}\right)-F_{+}\left(x_{j-1}\right)\right),
$$

with

$$
\left(F_{+}\left(x_{j}\right)-F_{+}\left(x_{j-1}\right)\right)=\int_{x_{j-1}}^{x_{j}} \nu(d x) .
$$

The approximation of the integral $\int_{x_{j-1}}^{x_{j}} \nu(d x)$ will depend on the Lévy measure. The quantities $\left(F_{+}\left(x_{k}\right)\right)_{1 \leq k \leq n}$ need be computed once.

We define the function $G_{+}$by, for any $y \in[0,1]$

$$
G_{+}(y)=x,
$$

where $x$ is the unique real satisfying

$$
\frac{F_{+}(x)}{F_{+}(A)}=y .
$$

Let $y \in[0,1]$, to compute $G_{+}(y)$, we use the following method. We have to find first the integer $k>1$ satifying $y_{k-1} \leq y<y_{k}$. Then we have

$$
y F_{+}(A)=y_{k-1}+\int_{x_{k-1}}^{G_{+}(y)} \nu(d y) .
$$

We must approximate the above integral depending on $G_{+}(y)$, and express the latter as a function of $y$. When $n$ and $A$ go to the infinity, $G_{+}(y)$ will converge to the inverse function of $F_{+}$. So we state that to simulate $Y_{1}^{+}$, we just need to simulate

$$
G_{+}(U) \text {, where } U \text { is a uniform r.v. on }[0,1] \text {. }
$$

5. Numerical examples. In this section we will first show an example of pricing using the method presented in this Section 4.

Consider a lookback call option with a predetermined maximum $S_{+}$. The arbitrage price is given by

$$
V\left(S_{+}\right)=e^{-r T} \mathbb{E}\left(S_{0} e^{M_{T}}-S_{0} e^{X_{T}}\right) .
$$

The process $X$ is a variance gamma process, i.e. for any $t \geq 0$

$$
X_{t}=\theta G_{t}+\sigma W_{G_{t}},
$$

where $W$ is a standard Brownian motion and $G$ is gamma process with parameter $(1 / \kappa, \kappa)$, i.e. the transition density of $G$ is given by

$$
f_{t}(x)=x^{\frac{t}{\kappa}-1} \frac{e^{-\frac{x}{\kappa}}}{\kappa^{\frac{t}{\kappa}} \Gamma\left(\frac{t}{\kappa}\right)}, x>0 .
$$

An approximation of $V\left(S_{+}\right)$is given by

$$
V^{\epsilon}\left(S_{+}\right)=e^{-r T} \mathbb{E}\left(S_{0} e^{M_{T}^{\epsilon}}-S_{0} e^{X_{T}^{\epsilon}}\right) .
$$


The resulting error is studied in Section 3. Note that for variance gamma processes the condition (2.6) is not satisfied.

To evaluate $V^{\epsilon}\left(S_{+}\right)$, we use the simulation method defined in Section 4. The parameters are $: S_{0}=100, r=0.0548, \delta=0, T=0.40504, S_{+}=100, \theta=-0.2859$, $\kappa=0.2505, \sigma=0.1927$ and the number of Monte Carlo simulations is $m=100000$. The number of discretization points on $[\epsilon, A]$ is $n=100000$ (see Section 4.3 for the definition of $A$ and $n$ ).

The fair price given by Becker (2010) is $V\left(S_{+}\right)=9.3982$. In Table 5.1, we compare $V\left(S_{+}\right)$and $V^{\epsilon}\left(S_{+}\right)$. The errors are relative to $V\left(S_{+}\right)$. The comptuing time

\begin{tabular}{|c|c|c|c|}
\hline$\epsilon$ & $V^{\epsilon}\left(S_{+}\right)$ & Monte Carlo error & Total error \\
\hline $10^{-1}$ & 7.076 & $0.05 \%$ & $24.7 \%$ \\
\hline $10^{-2}$ & 9.347 & $0.05 \%$ & $0.50 \%$ \\
\hline $10^{-3}$ & 9.401 & $0.05 \%$ & $0.04 \%$ \\
\hline
\end{tabular}

Approximated price of the continuous lookback call in VG model.

for $\epsilon=10^{-3}$ is less than 1 second. Note that we only use a standard Monte Carlo method.

We will, now, study numerically the optimality of the bounds derived in Section 3 . For example in Proposition 3.8, we have shown that (for the lookback call) we have

$$
\left|V\left(S_{-}\right)-V^{\epsilon}\left(S_{-}\right)\right| \leq C \sigma_{0}(\epsilon) .
$$

So

$$
\log \left(\left|V\left(S_{-}\right)-V^{\epsilon}\left(S_{-}\right)\right|\right) \leq \log (C)+\log \left(\sigma_{0}(\epsilon)\right) .
$$

Hence when $\epsilon$ goes to 0 , we will get

$$
\frac{\log \left(\left|V\left(S_{-}\right)-V^{\epsilon}\left(S_{-}\right)\right|\right)}{\log \left(\sigma_{0}(\epsilon)\right)} \geq \frac{\log (C)}{\log \left(\sigma_{0}(\epsilon)\right)}+1 .
$$

So we will represent in Figure 5.1 the function $f$ defined by

$$
f(\epsilon)=\frac{\log \left(\left|V\left(S_{-}\right)-V^{1-\epsilon}\left(S_{-}\right)\right|\right)}{\log \left(\sigma_{0}(1-\epsilon)\right)}, \forall \epsilon \in(0,1) .
$$

The function $f$ as expected converges to 1 when $\epsilon$ goes to 1 . The bound is, in the case of truncation, optimal for lookback options. For Brownian approximation this does not seem to be the case.

In Figure 5.2, we consider a lookback put. The function $f$ is this time defined by

$$
f(\epsilon)=\frac{\log \left(\left|V\left(S_{+}\right)-\hat{V}^{1-\epsilon}\left(S_{+}\right)\right|\right)}{\log (1-\epsilon)}, \forall \epsilon \in(0,1) .
$$

where $V\left(S_{+}\right)$is the price of the lookback put and $\hat{V}^{1-\epsilon}\left(S_{+}\right)$its Brownian approximation. The choice of the denominator in the definition of $f$ is due to the fact that we are looking for the best power of $\epsilon$ (actually $1-\epsilon$ in this case) that we can have. 


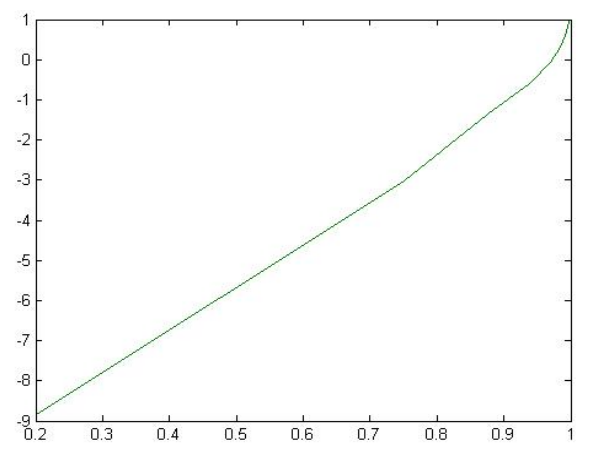

FIG. 5.1. Optimality of the error bound for a lookback call in VG model. The parameters are $S_{0}=100, r=0.0548, \delta=0, T=0.40504, S_{+}=100, \theta=-0.2859, \kappa=0.2505, \sigma=0.1927$.

We will compare this power with that obtained using the bounds in the second result of Proposition 3.9 (and in Proposition 3.3). In fact we will use the expression $\sigma_{0}(1-\epsilon) \beta(1-\epsilon)^{\frac{1}{5}} \log \left(\frac{1}{\beta(1-\epsilon)^{\frac{1}{5}}}\right)$ which is the best bound when $p$ goes to $+\infty$ in Proposition 3.9. Note that we do not pretend that $p$ can go to the infinity, but the bound in Proposition 3.9 cannot be better than the latter expression. In this expression, the power of $1-\epsilon$ in CGMY model is $1-0.3 Y$. The function $f$ converges to

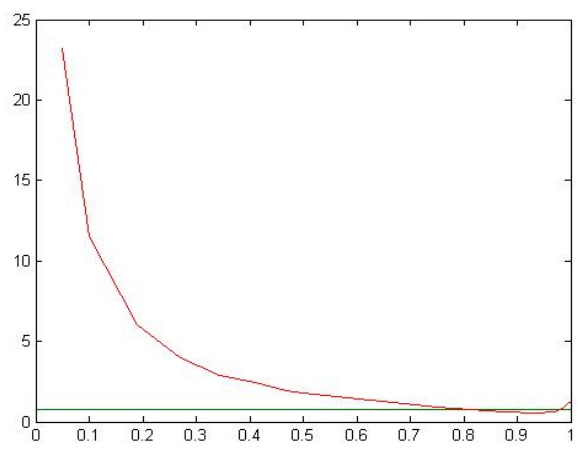

Fig. 5.2. Optimality of the error bound for a lookback put in CGMY model. The parameters are $S_{0}=100, r=0.05, \delta=0.02, T=1, S_{+}=100, C=4, G=50, M=60, Y=0.7$.

a constant bigger than $1-0.3 Y$ (which corresponds to the horizontal line) when $\epsilon$ goes to 1 . It seems that the function converges to $1+0.5 Y$ which corresponds to the bound $\sigma_{0}(1-\epsilon) \beta(1-\epsilon)$. We observe the same phenomenon with the Asian options.

For barrier options, we have the same remarks as for Asian and lookback option. The bound for barrier options seems to be optimal in jump truncation case. In Figure 5.3 we consider the case of a down and out call in VG model. So the function is defined by

$$
f(\epsilon)=\frac{\log \left(\left|V-V^{1-\epsilon}\right|\right)}{\log (1-\epsilon)}, \forall \epsilon \in(0,1)
$$


It converges to 1 when $\epsilon$ goes to 1 , as expected.

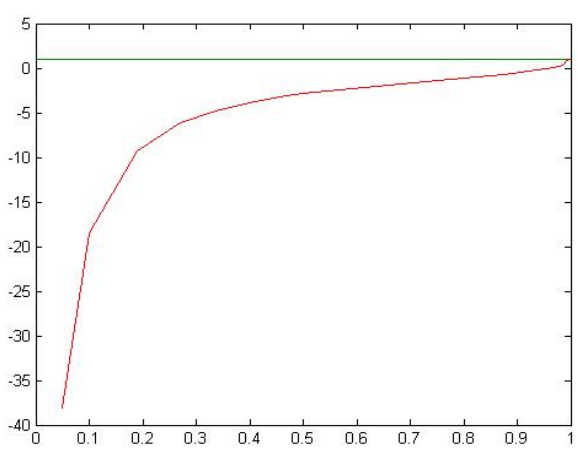

FiG. 5.3. Optimality of the error bound for a down and out call option in VG model. The parameters are $S_{0}=100, K=100, H=80, r=0.05, \delta=0.02, T=1, \theta=-0.2, \kappa=0.1, \sigma=0.16$.

\section{Appendix A.}

Proof of Lemma 4.2. We have

$$
\begin{aligned}
\left|\hat{M}_{T}^{\epsilon}-\bar{M}_{T}^{\epsilon}\right| & =\left|\max \left\{\max _{0 \leq j \leq N_{T}^{\epsilon}}\left\{\sup _{\hat{T}_{j}^{\epsilon} \leq s<\hat{T}_{j+1}^{\epsilon}} \hat{X}_{s}^{\epsilon}\right\}, \hat{X}_{T}^{\epsilon}\right\}-\max _{0 \leq j \leq N_{T}^{\epsilon}+1}\left\{\hat{X}_{\hat{T}_{j}^{\epsilon}}^{\epsilon}\right\}\right| \\
& \leq \max _{0 \leq j \leq N_{T}^{\epsilon}}\left\{\left|\sup _{\hat{T}_{j}^{\epsilon} \leq s<\hat{T}_{j+1}^{\epsilon}} \hat{X}_{s}^{\epsilon}-\hat{X}_{\hat{T}_{j}^{\epsilon}}^{\epsilon}\right|\right\} \\
& \leq \max _{0 \leq j \leq N_{T}^{\epsilon}}\left\{\sup _{\hat{T}_{j}^{\epsilon} \leq s<\hat{T}_{j+1}^{\epsilon}}\left|\hat{X}_{s}^{\epsilon}-\hat{X}_{\hat{T}_{j}^{\epsilon}}^{\epsilon}\right|\right\} \\
& \leq \sigma(\epsilon) \max _{0 \leq j \leq N_{T}^{\epsilon}}\left\{\sup _{\hat{T}_{j}^{\epsilon} \leq s<\hat{T}_{j+1}^{\epsilon}}\left|\hat{W}_{s}-\hat{W}_{\hat{T}_{j}^{\epsilon}}\right|\right\} .
\end{aligned}
$$

So

$$
\mathbb{E}\left|\hat{M}_{T}^{\epsilon}-\bar{M}_{T}^{\epsilon}\right|^{p} \leq \sigma(\epsilon)^{p} \mathbb{E} \max _{0 \leq j \leq N_{T}^{\epsilon}}\left\{\sup _{\hat{T}_{j}^{\epsilon} \leq s<\hat{T}_{j+1}^{\epsilon}}\left|\hat{W}_{s}-\hat{W}_{\hat{T}_{j}^{\epsilon}}\right|^{p}\right\} .
$$

Notice that, for $p \geq 1$

$$
\begin{aligned}
\max _{0 \leq j \leq N_{T}^{\epsilon}}\left\{\sup _{\hat{T}_{j}^{\epsilon} \leq s<\hat{T}_{j+1}^{\epsilon}}\left|\hat{W}_{s}-\hat{W}_{\hat{T}_{j}^{\epsilon}}\right|^{p}\right\} & \leq 2^{p-1} \max _{0 \leq j \leq N_{T}^{\epsilon}}\left\{\sup _{\hat{T}_{j}^{\epsilon} \leq s<\hat{T}_{j+1}^{\epsilon}}\left|\hat{W}_{s}\right|^{p}+\left|\hat{W}_{\hat{T}_{j}^{\epsilon}}\right|^{p}\right\} \\
& \leq 2^{p} \max _{0 \leq j \leq N_{T}^{\epsilon}}\left\{\sup _{\hat{T}_{j}^{\epsilon} \leq s<\hat{T}_{j+1}^{\epsilon}}\left|\hat{W}_{s}\right|^{p}\right\} \\
& \leq 2^{p} \sup _{0 \leq s \leq T}\left|\hat{W}_{s}\right|^{p} .
\end{aligned}
$$


On the other hand

$$
\lim _{\epsilon \rightarrow 0}\left(\hat{T}_{j+1}^{\epsilon}-\hat{T}_{j}^{\epsilon}\right)=0, \text { a.s. }
$$

So by dominated convergence

$$
\lim _{\epsilon \rightarrow 0} \mathbb{E} \max _{0 \leq j \leq N_{T}^{\epsilon}}\left\{\sup _{\hat{T}_{j}^{\epsilon} \leq s<\hat{T}_{j+1}^{\epsilon}}\left|\hat{W}_{s}-\hat{W}_{\hat{T}_{j}^{\epsilon}}\right|^{p}\right\}=0 .
$$

This concludes the proof. $\square$

Proof of Proposition 4.3. Set

$$
Z^{\epsilon}=\frac{1}{T} \int_{0}^{T} S_{0} e^{\hat{X}_{s}^{\epsilon}} d s
$$

We have

$$
\begin{aligned}
Z^{\epsilon} & =\frac{S_{0}}{T} \sum_{j=1}^{N_{T}^{\epsilon}+1} e^{\sum_{i=1}^{j-1} Y_{i}^{\epsilon}} \int_{\hat{T}_{j-1}^{\epsilon}}^{\hat{T}_{j}^{\epsilon}} e^{\gamma_{0}^{\epsilon} s+\sigma(\epsilon) \hat{W}_{s}} d s \\
& =\frac{S_{0}}{T} \sum_{j=1}^{N_{T}^{\epsilon}+1} e^{\sum_{i=1}^{j-1} Y_{i}^{\epsilon}+\gamma_{0}^{\epsilon} \hat{T}_{j-1}^{\epsilon}+\sigma(\epsilon) \hat{W}_{\hat{T}_{j-1}^{\epsilon}}} \int_{\hat{T}_{j-1}^{\epsilon}}^{\hat{T}_{j}^{\epsilon}} e^{\gamma_{0}^{\epsilon}\left(s-\hat{T}_{j-1}^{\epsilon}\right)+\sigma(\epsilon)\left(\hat{W}_{s}-\hat{W}_{\hat{T}_{j-1}^{\epsilon}}\right)} d s \\
& =\frac{S_{0}}{T} \sum_{j=1}^{N_{T}^{\epsilon}+1} e^{\hat{X}_{\hat{T}_{j-1}^{\epsilon}}^{\epsilon}} \int_{\hat{T}_{j-1}^{\epsilon}}^{\hat{T}_{j}^{\epsilon}} e^{\gamma_{0}^{\epsilon}\left(s-\hat{T}_{j-1}^{\epsilon}\right)+\sigma(\epsilon)\left(\hat{W}_{s}-\hat{W}_{\hat{T}_{j-1}^{\epsilon}}\right)} d s .
\end{aligned}
$$

Note that using the integral form of Taylor Theorem, we have

$$
\begin{aligned}
e^{\sigma(\epsilon)\left(\hat{W}_{s}-\hat{W}_{\hat{T}_{j-1}^{\epsilon}}\right)=}=1+\sigma(\epsilon)\left(\hat{W}_{s}-\hat{W}_{\hat{T}_{j-1}^{\epsilon}}\right) \\
+\int_{0}^{\sigma(\epsilon)\left(\hat{W}_{s}-\hat{W}_{\hat{T}_{j-1}^{\epsilon}}\right)} e^{y}\left(\sigma(\epsilon)\left(\hat{W}_{s}-\hat{W}_{\hat{T}_{j-1}^{\epsilon}}\right)-y\right) d y
\end{aligned}
$$

So, noting $\alpha$ the Lipschitz constant of $f$, we have

$$
\begin{aligned}
\delta_{\epsilon}:= & \mathbb{E} f\left(\frac{1}{T} \int_{0}^{T} S_{0} e^{\hat{X}_{s}^{\epsilon}} d s\right)-\mathbb{E} f\left(\frac{S_{0}}{T} \sum_{j=1}^{N_{T}^{\epsilon}+1} e^{\hat{X}_{\hat{T}_{j-1}^{\epsilon}}^{\epsilon}}\left(\frac{e^{\gamma_{0}^{\epsilon}\left(\hat{T}_{j}^{\epsilon}-\hat{T}_{j-1}^{\epsilon}\right)}-1}{\gamma_{0}^{\epsilon}}+\sigma(\epsilon) g_{j}^{\epsilon}\right)\right) \mid \\
\leq & \alpha \mathbb{E} \frac{S_{0}}{T} \sum_{j=1}^{N_{T}^{\epsilon}+1} e^{\hat{X}_{\hat{T}_{j-1}^{\epsilon}}^{\epsilon}} \int_{\hat{T}_{j-1}^{\epsilon}}^{\hat{T}_{j}^{\epsilon}} d s e^{\gamma_{0}^{\epsilon}\left(s-\hat{T}_{j-1}^{\epsilon}\right)} \\
& \times\left|\int_{0}^{\sigma(\epsilon)\left(\hat{W}_{s}-\hat{W}_{\hat{T}_{j-1}^{\epsilon}}\right)} e^{y}\left(\sigma(\epsilon)\left(\hat{W}_{s}-\hat{W}_{\hat{T}_{j-1}^{\epsilon}}\right)-y\right) d y\right| \\
\leq & \alpha \frac{\sigma(\epsilon)^{2}}{2} \frac{S_{0}}{T} \mathbb{E} \sum_{j=1}^{N_{T}^{\epsilon}+1} e^{\hat{X}_{\hat{T}_{j-1}^{\epsilon}}^{\epsilon}} \int_{\hat{T}_{j-1}^{\epsilon}}^{\hat{T}_{j}^{\epsilon}}\left(\hat{W}_{s}-\hat{W}_{\hat{T}_{j-1}^{\epsilon}}\right)^{2} e^{\gamma_{0}^{\epsilon}\left(s-\hat{T}_{j-1}^{\epsilon}\right)+\sigma(\epsilon)\left(\hat{W}_{s}-\hat{W}_{\hat{T}_{j-1}^{\epsilon}}\right)^{+}} d s .
\end{aligned}
$$


Notice that for $s$ in $\left[\hat{T}_{j-1}^{\epsilon}, \hat{T}_{j}^{\epsilon}\right]$, we have

$$
\begin{aligned}
\hat{X}_{\hat{T}_{j-1}^{\epsilon}}^{\epsilon}+\gamma_{0}^{\epsilon}\left(s-\hat{T}_{j-1}^{\epsilon}\right)+\sigma(\epsilon)\left(\hat{W}_{s}-\hat{W}_{\hat{T}_{j-1}^{\epsilon}}\right)^{+}= & \sum_{i=1}^{j-1} Y_{i}^{\epsilon}+\gamma_{0}^{\epsilon} s+\sigma(\epsilon) \hat{W}_{\hat{T}_{j-1}^{\epsilon}} \\
& +\sigma(\epsilon)\left(\hat{W}_{s}-\hat{W}_{\hat{T}_{j-1}^{\epsilon}}\right)^{+} \\
\leq & \sum_{i=1}^{j-1} Y_{i}^{\epsilon}+\gamma_{0}^{\epsilon} s+\sigma(\epsilon) \sup _{\hat{T}_{j-1}^{\epsilon} \leq \tau \leq \hat{T}_{j}^{\epsilon}} \hat{W}_{\tau} \\
\leq & \sum_{i=1}^{j-1} Y_{i}^{\epsilon}+\gamma_{0}^{\epsilon} s+\sigma(\epsilon) \sup _{0 \leq \tau \leq T} \hat{W}_{\tau} .
\end{aligned}
$$

Thus

$$
\begin{aligned}
\delta_{\epsilon} \leq & \alpha \frac{\sigma(\epsilon)^{2}}{2} \frac{S_{0}}{T} \mathbb{E} \sum_{j=1}^{N_{T}^{\epsilon}+1} e^{\sum_{i=1}^{j-1} Y_{i}^{\epsilon}} \int_{\hat{T}_{j-1}^{\epsilon}}^{\hat{T}_{j}^{\epsilon}} d s e^{\gamma_{0}^{\epsilon} s}\left(\sup _{0 \leq \tau \leq T} \hat{W}_{\tau}-\inf _{0 \leq \tau \leq T} \hat{W}_{\tau}\right)^{2} \\
& \times e^{\sigma(\epsilon) \sup _{0 \leq \tau \leq T} \hat{W}_{\tau}} \\
\leq & \alpha \frac{\sigma(\epsilon)^{2}}{2} \frac{S_{0}}{T} \mathbb{E} \sum_{j=1}^{N_{T}^{\epsilon}+1} e^{\sum_{i=1}^{j-1} Y_{i}^{\epsilon}}\left(e^{\gamma_{0}^{\epsilon} \hat{T}_{j}^{\epsilon}}+e^{\gamma_{0}^{\epsilon} \hat{T}_{j-1}^{\epsilon}}\right) \\
& \times \int_{\hat{T}_{j-1}^{\epsilon}}^{\hat{T}_{j}^{\epsilon}} d s \mathbb{E}\left(\sup _{0 \leq \tau \leq T} \hat{W}_{\tau}-\inf _{0 \leq \tau \leq T} \hat{W}_{\tau}\right)^{2} e^{\sigma(\epsilon) \sup _{0 \leq \tau \leq T} \hat{W}_{\tau}} \\
\leq & \alpha \frac{\sigma(\epsilon)^{2}}{2} \frac{S_{0}}{T} \mathbb{E} \sum_{j=1}^{N_{T}^{\epsilon}+1}\left(e^{X_{\hat{T}_{j}^{\epsilon-}}^{\epsilon}}+e^{X_{\dot{T}_{j-1}^{\epsilon}}^{\epsilon}}\right)\left(\hat{T}_{j}^{\epsilon}-\hat{T}_{j-1}^{\epsilon}\right) \mathbb{E}\left(\sup _{0 \leq \tau \leq T} \hat{W}_{\tau}-\inf _{0 \leq \tau \leq T} \hat{W}_{\tau}\right)^{2} \\
& \times e^{\sigma(\epsilon) \sup _{0 \leq \tau \leq T} \hat{W}_{\tau}} .
\end{aligned}
$$

So

$$
\begin{aligned}
\delta_{\epsilon} & \leq \alpha \sigma(\epsilon)^{2} \frac{S_{0}}{T} \mathbb{E} \sum_{j=1}^{N_{T}^{\epsilon}+1} e^{M_{T}^{\epsilon}}\left(\hat{T}_{j}^{\epsilon}-\hat{T}_{j-1}^{\epsilon}\right) \mathbb{E}\left(\sup _{0 \leq \tau \leq T} \hat{W}_{\tau}-\inf _{0 \leq \tau \leq T} \hat{W}_{\tau}\right)^{2} e^{\sigma(\epsilon) \sup _{0 \leq \tau \leq T} \hat{W}_{\tau}} \\
& \leq \alpha \sigma(\epsilon)^{2} S_{0} \mathbb{E} e^{M_{T}^{\epsilon}} \mathbb{E}\left(\sup _{0 \leq \tau \leq T} \hat{W}_{\tau}-\inf _{0 \leq \tau \leq T} \hat{W}_{\tau}\right)^{2} e^{\sigma(\epsilon) \sup _{0 \leq \tau \leq T} \hat{W}_{\tau}} .
\end{aligned}
$$

Using Lemma 1 of [9], we conclude that

$$
\delta_{\epsilon}=O\left(\sigma(\epsilon)^{2}\right) .
$$

Proof of Proposition 4.4. We have

$$
\begin{aligned}
\delta_{\epsilon} & :=\mathbb{E} \int_{0}^{T} e^{X_{s}} d s-\mathbb{E} \int_{0}^{T} e^{X_{s}^{\epsilon}} d s \\
& =\int_{0}^{T} \mathbb{E}\left(e^{X_{s}}-e^{X_{s}^{\epsilon}}\right) d s .
\end{aligned}
$$


Using the integral form of the Taylor Theorem, we get

$$
\begin{aligned}
\mathbb{E}\left(e^{X_{s}}-e^{X_{s}^{\epsilon}}\right) & =\frac{\sigma(\epsilon)^{2} s}{2} \mathbb{E} e^{X_{s}^{\epsilon}}+\int_{0}^{1} \mathbb{E}\left(e^{X_{s}^{\epsilon}+\theta R_{s}^{\epsilon}}-e^{X_{s}^{\epsilon}}\right)(1-\theta)\left(R_{s}^{\epsilon}\right)^{2} d \theta \\
& =\frac{\sigma(\epsilon)^{2} s}{2} \mathbb{E} e^{X_{s}^{\epsilon}}+\mathbb{E} e^{X_{s}^{\epsilon}}\left(\mathbb{E} e^{R_{s}^{\epsilon}}-1-R_{s}^{\epsilon}-\frac{\left(R_{s}^{\epsilon}\right)^{2}}{2}\right) .
\end{aligned}
$$

The last equality is obtained by a simple integration. On the other hand

$$
\begin{aligned}
\mathbb{E} e^{X_{s}^{\epsilon}}\left|\mathbb{E} e^{R_{s}^{\epsilon}}-1-R_{s}^{\epsilon}-\frac{\left(R_{s}^{\epsilon}\right)^{2}}{2}\right| & \leq \mathbb{E} e^{X_{s}^{\epsilon}} \mathbb{E}\left|R_{s}^{\epsilon}\right|^{3} e^{\sup _{0 \leq u \leq s} R_{u}^{\epsilon}} \\
& \leq \mathbb{E} e^{X_{s}^{\epsilon}}\left(\mathbb{E}\left|R_{s}^{\epsilon}\right|^{4}\right)^{\frac{3}{4}}\left(\mathbb{E} e^{4 \sup _{0 \leq u \leq s} R_{u}^{\epsilon}}\right)^{\frac{1}{4}} \\
& \leq \mathbb{E} e^{X_{s}^{\epsilon}}\left(\mathbb{E}\left|R_{s}^{\epsilon}\right|^{4}\right)^{\frac{3}{4}}\left(\mathbb{E} e^{4 \sup _{0 \leq u \leq T} R_{u}^{\epsilon}}\right)^{\frac{1}{4}} .
\end{aligned}
$$

Using Proposition 6 of [10], we conclude that

$$
\mathbb{E} e^{X_{s}^{\epsilon}}\left|\mathbb{E} e^{R_{s}^{\epsilon}}-1-R_{s}^{\epsilon}-\frac{\left(R_{s}^{\epsilon}\right)^{2}}{2}\right|=O\left(\sigma_{0}(\epsilon)^{3}\right) .
$$

uniformly with $s \in[0, T]$. Hence

$$
\mathbb{E} \int_{0}^{T} e^{X_{s}} d s-\mathbb{E} \int_{0}^{T} e^{X_{s}^{\epsilon}} d s=\frac{\sigma(\epsilon)^{2}}{2} \int_{0}^{T} s \mathbb{E} e^{X_{s}^{\epsilon}} d s+O\left(\sigma_{0}(\epsilon)^{3}\right) .
$$

Replacing in the previous expressions $X_{s}$ by $\hat{X}_{s}^{\epsilon}$, we will obtain $\sigma(\epsilon) \hat{W}_{s}$ instead of $R_{s}^{\epsilon}$. Thus

$$
\mathbb{E} \int_{0}^{T} e^{\hat{X}_{s}^{\epsilon}} d s-\mathbb{E} \int_{0}^{T} e^{X_{s}^{\epsilon}} d s=\frac{\sigma(\epsilon)^{2}}{2} \int_{0}^{T} s \mathbb{E} e^{X_{s}^{\epsilon}} d s+O\left(\sigma_{0}(\epsilon)^{3}\right) .
$$

Therefore

$$
\mathbb{E} \int_{0}^{T} e^{X_{s}} d s-\mathbb{E} \int_{0}^{T} e^{\hat{X}_{s}^{\epsilon}} d s=O\left(\sigma_{0}(\epsilon)^{3}\right)
$$

\section{REFERENCES}

[1] Asmussen, S \& Rosinski, J 2001, 'Approximations of small jumps of Lévy processes with a view towards simulation', J. Appl. Probab., vol. 38, pp. 482-493.

[2] Barndorff-Nielsen, OE 1996, 'Normal Inverse Gaussian Processes and the Modelling of Stock Returns', Scand. J. Statistics, vol. 24, pp. 1-13.

[3] Becker, M 2010, 'Unbiased Monte Carlo Valuation of Lookback, Swing and Barrier Options Under Variance Gamma Model', Journal of Computational Finance, vol. 13, no. 4.

[4] Broadie, M, \& Yamamoto, Y 2005, 'A double-exponential fast Gauss transform algorithm for pricing discrete path-dependent options', Operations Research, vol. 53, pp. 764-779.

[5] Carr, P. P., Geman, H, Madan DB \& Yor, M 2002, 'The fine structure of asset returns: An empirical investigation', Journal of Business, vol .75, pp. 305-332.

[6] Chaumont, L 2010, 'On the law of the supremum of Lévy processes', submitted for publication. 
[7] Cont, R \& Tankov, P 2004, Financial modelling with jump processes, Chapman \& Hall/CRC Financial Mathematics Series, Boca Raton.

[8] Cont, R \& Voltchkova, E 2005, 'Integro-differential equations for option prices in exponential Lévy models', Finance Stochast., vol. 3, pp. 299-325.

[9] Dia, EHA 2010, 'Exotic Options under Exponential Lévy Model', Doctoral thesis, Université Paris-Est, http://tel.archives-ouvertes.fr/INSMI/tel-00520583/fr/.

[10] Dia, EHA 2010, 'Error bounds for small jumps of Lévy processes', Advances in Applied Probabiliy, Forthcoming 2013.

[11] Eberlein, E 2001, 'Application of generalized hyperbolic Lévy motions to finance', in Lévy Processes: Theory and Applications, eds OE Barndorff-Nielsen, T Mikosch \& S Resnick, Birkhäuser Verlag, pp. 319-337.

[12] Feng, L \& Linetsky, V 2009, 'Computing Exponential Moments of the Discrete Maximum of a Lévy process and Lookback Options', Finance and Stochastics vol. 13, no. 4, pp. 501-529.

[13] Feng, L \& Linetsky, V 2008, 'Pricing Discretely Monitored Barrier Options and Defaultable Bonds in Lévy Process Models: a Fast Hilbert Transform Approach', Mathematical Finance, vol. 18 , no. 3, pp. 337-384.

[14] Kohatsu-Higa, A \& Tankov, P 2010, 'Jump-adapted discretization schemes for Lévy-driven SDEs', Stochastic Processes and their Applications, vol. 120, no. 11, pp. 2258-2285.

[15] Madan, DB, Carr, PP \& Chang, EC 1998, 'The Variance Gamma Process and Option Pricing', European Finance Review, vol. 2, no. 1, pp. 79-105.

[16] Kou, SG \& Petrella, G 2004, 'Numerical Pricing of Discrete Barrier and Lookback Options via Laplace Transforms' Journal of Computational Finance, vol. 8, pp. 1-37.

[17] Rydberg, TH 1997, 'The normal inverse gaussian lévy process: simulation and approximation', Stochastic Models, vol. 13, no. 4, pp. 887-910.

[18] Sato, K 1999 Lévy processes and infinitely divisible distributions, Cambridge university press.

[19] Signahl, M 2003, 'On error rates in Normal approximations and simulation schemes for Lévy processes', Stoch. Models., vol. 19, pp. 287-298.

[20] Skorokhod, AV 1965, Studies in the Theory of Random Processes, Reading, Mass., AddisonWesley - cop.

[21] Taufer, E \& Leonenko, N 2009, 'Simulation of Lévy-driven Ornstein-Uhlenbeck processes with given marginal distribution' Comput. Statist. Data Anal., vol. 53, no. 6, pp. 2427-2437.

[22] Todorov, V \& Tauchen, G 2006, 'Simulation methods for Lévy-driven continuous-time autoregressive moving average (CARMA) stochastic volatility models', J. Bus. Econom. Statist. vol. 24 , no. 4 , pp. $455-469$ 